\title{
LOCAL SINGLE RING THEOREM
}

\author{
By FLORENT BENAYCH-GEORGES
}

\section{Université Paris Descartes}

The single ring theorem, by Guionnet, Krishnapur and Zeitouni in Ann. of Math. (2) 174 (2011) 1189-1217, describes the empirical eigenvalue distribution of a large generic matrix with prescribed singular values, that is, an $N \times N$ matrix of the form $A=U T V$, with $U, V$ some independent Haardistributed unitary matrices and $T$ a deterministic matrix whose singular values are the ones prescribed. In this text, we give a local version of this result, proving that it remains true at the microscopic scale $(\log N)^{-1 / 4}$. On our way to prove it, we prove a matrix subordination result for singular values of sums of non-Hermitian matrices, as Kargin did in Ann. Probab. 43 (2015) 21192150 for Hermitian matrices. This allows to prove a local law for the singular values of the sum of two non-Hermitian matrices and a delocalization result for singular vectors.

Introduction. The single ring theorem, by Guionnet, Krishnapur and Zeitouni in [22], describes the empirical eigenvalue distribution of a large generic matrix with prescribed singular values, that is, an $N \times N$ matrix of the form $A=U T V$, with $U, V$ some independent Haar-distributed unitary matrices and $T$ a deterministic matrix whose singular values are the ones prescribed. More precisely, under some technical hypotheses, as the dimension $N$ tends to infinity, if the empirical distribution of the singular values of $A$ (i.e., of $T$ ) converges to a compactly supported limit probability measure $v$ on the real line, then the empirical eigenvalue distribution of $A$ converges to a limit probability measure $\mu$ on the complex plane which depends only on $v$. The limit measure $\mu$ is radial, has support

$$
S:=\{z \in \mathbb{C} ; a \leq|z| \leq b\} \quad \text { for } a^{-2}:=\int x^{-2} \mathrm{~d} v(x) ; b^{2}:=\int x^{2} \mathrm{~d} v(x)
$$

and density $\rho$ satisfying

$$
\rho(z):=\frac{1}{2 \pi} \Delta_{z}\left(\int \log |x| v_{\infty, z}(\mathrm{~d} x)\right) \quad \text { with } v_{\infty, z}:=v^{s} \boxplus \frac{\delta_{|z|}+\delta_{-|z|}}{2}
$$

( $v^{s}$ is the symmetrization of $v$, see (10), and $\boxplus$ is the additive free convolution $[1,30,33])$. In the left image of Figure 1, we plotted the spectrum of an example of such a matrix $A$ with size $N=500$, illustrating the convergence of the

Received February 2015; revised April 2016.

MSC2010 subject classifications. 15B52, 60B20, 46L54.

Key words and phrases. Random matrices, single ring theorem, local laws, free convolution, free probability theory, haar measure. 


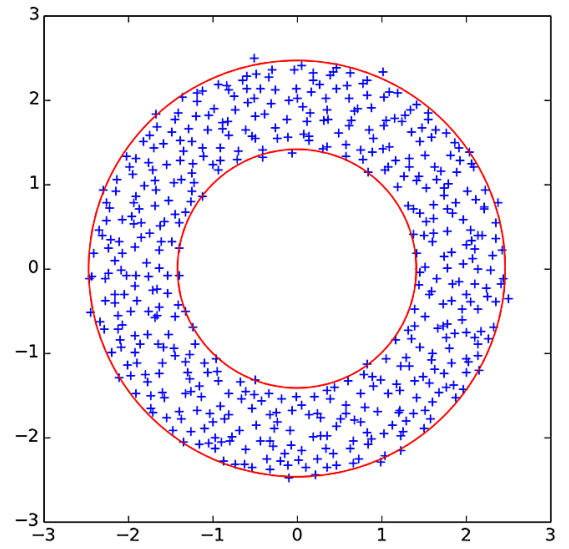

(a)

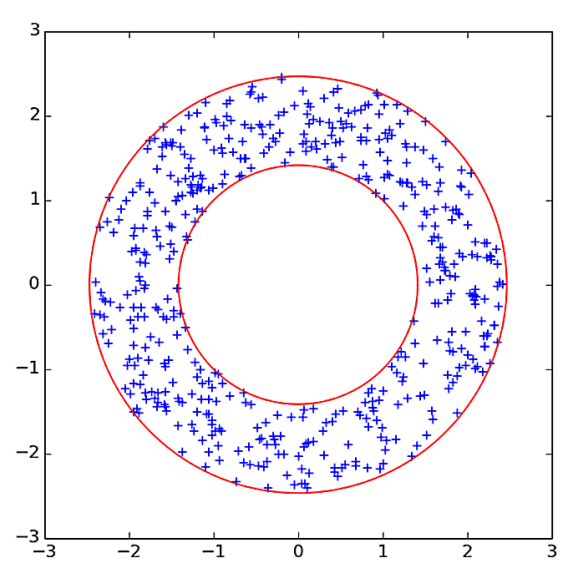

(b)

FIG. 1. Repulsion (eigenvalues of A)/lack of repulsion (independently distributed points). (a) Spectrum of the $500 \times 500$ matrix $A=U T V$ when the singular values of $T$ are uniformly distributed on $(0.5,4)$. (b) 500 points uniformly distributed on the support of the limit spectral distribution of $A$.

empirical spectral measure. In the right image of Figure 1, we plotted 500 independent random points with uniform distribution on the ring $S$. Our point was not to compare the limit spectral distribution of $A$ with the uniform distribution on $S$, but to compare both point processes at microscopic scale: we see that the 500 eigenvalues of $A$ fill the ring way more regularly than the independent points, which reflects the so-called eigenvalues repulsion phenomenon. Some of the mathematical manifestations of such phenomenons are the so-called local laws (see, e.g., $[16,17,19])$. Here, we will prove a local law for the single ring theorem on scale $\varepsilon_{N}=(\log N)^{-1 / 4+\epsilon}$ in the interior of $S$, which means roughly that the number of eigenvalues of $A$ in any ball $B\left(z_{0}, r\right)$ contained in $S$ is asymptotic to $\mu\left(B\left(z_{0}, r\right)\right) \times N$ not only for fixed $r$ but also for $r \sim \varepsilon_{N}$.

To give an idea of the techniques used in the proofs and of the difficulties we had to overcome, let us compare them with those of another local law for nonHermitian matrices. Recently, in the series of papers [16, 17, 34], Bourgade, Yau and Yin proved a local law for non-Hermitian matrices with i.i.d. entries. It is well known that the empirical spectral distribution of a random matrix with size $N$ whose entries are i.i.d., centered, with variance $1 / N$ and subject to no symmetry tends to the uniform measure on the unit disc of $\mathbb{C}$ when the dimension tends to infinity (see [32]). In [16, 17, 34], the authors gave an almost optimal result about the local accuracy of the approximation of the empirical spectral distribution by its limit: they proved, through $\mathcal{C}^{2}$ test functions, that the approximation stays correct as long as we consider test sets with surface at least $N^{\epsilon-1}$, for any $\epsilon>0$. As a subset of the unit disc with normalized surface $\mathcal{S}$ should contain approximately $N \times \mathcal{S}$ eigenvalues, this is not far from the best one could do by considering sets with more than finitely many eigenvalues. In the local law we give here, we are 
far from this optimal scale, but the set of tools we have at disposal lacks several key elements. The proofs, in $[16,17,34]$ as well as in the present paper, are based on the so-called Hermitization technique, which expresses the empirical spectral distribution of a non-Hermitian matrix $A$ as the Laplacian of the function $f(z)=$ $\frac{1}{N} \operatorname{Tr} \log |A-z|$, with $|A-z|=\sqrt{(A-z)(A-z)^{*}}$ [see (45)]. In $[16,17,34]$, where $A$ is a matrix with i.i.d. entries, $A-z$ is a matrix of the type "information plus noise", a model well understood. It allows the authors of $[16,17,34]$ to prove, thanks to the Schur complements formula, that for any $z$, the empirical eigenvalues distribution of $|A-z|$ is close to its limit at local scale $N^{\epsilon-1}$. Then, as the limit spectral distribution of $|A-z|$ has a smooth density whose singularity points are well understood and as the smallest singular values of $z-A$ are not likely to be too close to zero, the authors of $[16,17,34]$ approximate $\frac{1}{N} \operatorname{Tr} \log |A-z|$ by its theoretical limit quite well. Here, the Schur complements formula is not an option because suppressing a row and a column breaks the symmetry of the Haar measure. Instead, we use the matrix subordination, a technique proposed by Kargin in [28]: in Theorem 1.5, we prove that for any matrix $B$ independent of $A=U T V$, the resolvants $G_{\mathbf{A}}, G_{\mathbf{B}}$ and $G_{\mathbf{H}}$ of the matrices

$$
\mathbf{A}:=\left(\begin{array}{cc}
0 & A \\
A^{*} & 0
\end{array}\right) ; \quad \mathbf{B}:=\left(\begin{array}{cc}
0 & B \\
B^{*} & 0
\end{array}\right) \quad \text { and } \quad \mathbf{H}:=\left(\begin{array}{cc}
0 & A+B \\
(A+B)^{*} & 0
\end{array}\right)
$$

at $z=E+\mathrm{i} \eta, \eta \geq N^{1 / 8}$, satisfy

(3) $\mathbb{E} G_{\mathbf{H}}(z)=G_{\mathbf{A}}\left(z+S_{B}(z)\right)+\left(\right.$ error term with operator norm $\left.\leq \frac{1}{N \eta^{6}}\right)$,

(4) $\mathbb{E} G_{\mathbf{H}}(z)=G_{\mathbf{B}}\left(z+S_{A}(z)\right)+\left(\right.$ error term with operator norm $\left.\leq \frac{1}{N \eta^{6}}\right)$

for some complex-valued functions $S_{A}, S_{B}$ such that $\operatorname{Im} S_{A, B}(z) \geq-\frac{1}{N \eta^{7}}$. Equations (3) and (4) have to be compared with the ones defining the free convolution $\boxplus$ thanks to Stieltjes' transforms subordination (see Theorem A.10):

$$
\begin{array}{ll}
m_{\mu^{s} \boxplus v^{s}}(z)=m_{\nu^{s}}\left(z+S_{\mu}(z)\right) ; & \operatorname{Im} S_{v}(z) \geq 0, \\
m_{\mu^{s} \boxplus \nu^{s}}(z)=m_{\mu^{s}}\left(z+S_{v}(z)\right) ; & \operatorname{Im} S_{v}(z) \geq 0 .
\end{array}
$$

The Hermitization technique described above brings us to use these equations with $B=-z I$ and $\mu=\delta_{|z|}$, so that $\mu^{s} \boxplus v^{s}=v_{\infty, z}$. Ideally, equations (3)-(6) should give an upper bound on $\mathbb{E} \frac{1}{2 N} \operatorname{Tr} G_{\mathbf{H}}(z)-m_{\mu^{s} \boxplus v^{s}}(z)$ which could be turned into an upper bound on

$$
\frac{1}{2 N} \operatorname{Tr} \log |\mathbf{H}|-\int \log |x| \mathrm{d} \mu^{s} \boxplus v^{s}(x) .
$$

The problem here is that the upper bound on $\mathbb{E} \frac{1}{N} \operatorname{Tr} G_{\mathbf{H}}(z)-m_{\mu^{s} \boxplus v^{s}}(z)$ deduced from equations (3)-(6) involves the inverse of a certain $2 \times 2$ determinant [see 
(31)], which can vanish for $z$ close to the real line (to control this determinant for $z$ close to the real line, one would need precise informations about the density of $v_{\infty, z}$, which have, except for the case of i.i.d. matrices, remained out of reach so far, despite several studies of these questions as in $[5,7,9]) .{ }^{1}$ However, using only some bounds on the operator norms of $A$ and $B$, we can deduce from (3)-(6) that for $|z|$ large enough,

$$
\left|\mathbb{E} \frac{1}{N} \operatorname{Tr} G_{\mathbf{H}}(z)-m_{\mu^{s} \boxplus \nu^{s}}(z)\right| \leq \frac{C}{N} .
$$

The necessity to have $|z|$ large for such a bound to be proved is a real problem in the perspective of establishing a local law for the eigenvalues of $A$. We fix it (at the price of a quite poor microscopic scale $\varepsilon_{N}$ ) using Hadamard's three circles theorem, an idea introduced by Kargin in [26]. This theorem, with some standard concentration inequalities, allows to deduce from (8) that for $\eta \sim \frac{1}{\sqrt{\log N}}$, we have

$$
\frac{1}{N} \operatorname{Tr} G_{\mathbf{H}}(z)-m_{\mu^{s} \boxplus v^{s}}(z) \ll 1
$$

To complete the proof, we need to turn (9) into a control on (7): this is done thanks to the Helffer-Sjőstrand functional calculus and to a recent theorem by Rudelson and Vershynin in [31] about the smallest singular value of $A-z$.

The recent preprints $[2,3]$ by Bao, Erdős and Schnelli give local laws for the close model $A+U B U^{*}$ when $\mu_{A} \rightarrow \mu_{\alpha}$ and $\mu_{B} \rightarrow \mu_{\beta}$ as $N \rightarrow \infty$. Their local laws are established at some better scales than the ones we give here for $U T V^{*}-z$, but, seemingly facing the same problem as us, they had to specify the part $\mathcal{B}_{\mu_{\alpha} \boxplus \mu_{\beta}}$ of the real line where they establish these laws, avoiding a set of singular points (see [2], Theorem 2.7, and [3], Theorem 2.5). It should be possible to adapt their proofs to our model $U T V^{*}-z$, but at the current level of understanding of the densities of the laws $v_{\infty, z}$, we do not know exactly what the sets $\mathcal{B}_{v_{\infty, z}}$ look like and how to deal with their complementaries, thus it is today not possible to convert such local laws into a local version of the single ring theorem.

Organization of the article: We postpone the proof of our key result, the matrix subordination result (Theorem 1.5) to Section 5. We will first prove its main consequence, Proposition 2.1, in Section 2. Then the short proofs of the local law for the singular values of $A+\widetilde{B}$ (Theorem 1.10) and of the singular vectors delocalization for $A+\widetilde{B}$ (Theorem 1.12) will be given in Section 3. The proof of the local single ring theorem will be given in Section 4, followed in Section 5 by the proof of Theorem 1.5 and in the Appendix by several results we will use here.

\footnotetext{
${ }^{1}$ The lack of information on the order of the density of $v_{\infty, z}$ at its singularities is also what makes the use of the estimates of Guionnet, Krishnapur and Zeitouni, like [23], equation (12), ineffective here.
} 
Notation: Throughout this text, $z=E+\mathrm{i} \eta, E \in \mathbb{R}, \eta>0$ denotes an element of $\mathbb{C}^{+}:=\{\xi \in \mathbb{C} ; \operatorname{Im} \xi>0\}$. For $\mu$ a signed measure on the real line, we define

$$
\begin{aligned}
& \mu^{s}(X):=\frac{1}{2}(\mu(X)+\mu(-X)) \quad(\text { symmetrization of } \mu), \\
& m_{\mu}(z):=\int \frac{\mathrm{d} \mu(t)}{t-z} \quad(\text { Stieltjes transform of } \mu)
\end{aligned}
$$

and for $M \in \mathcal{M}_{N}(\mathbb{C})$ (the set of $N \times N$ complex matrices), we define $\mu_{M}$ as the empirical eigenvalue distribution of $M$ :

$$
\mu_{M}:=\frac{1}{N} \sum_{i=1}^{N} \delta_{\lambda_{i}} \quad\left(\lambda_{1}, \ldots, \lambda_{N}: \text { eigenvalues of } M\right),
$$

whereas $v_{M}$ denotes the empirical singular value distribution of $M$ :

$$
v_{M}:=\frac{1}{N} \sum_{i=1}^{N} \delta_{s_{i}} \quad\left(s_{1}, \ldots, s_{N}: \text { singular values of } M\right) .
$$

Note that we have

$$
v_{M}^{s}=\mu_{\mathbf{M}} \quad \text { for } \mathbf{M}:=\left(\begin{array}{cc}
0 & M \\
M^{*} & 0
\end{array}\right)
$$

We denote by $\|M\|$ the canonical operator norm of $M$. When $M$ is Hermitian, we also define, for $z=E+\mathrm{i} \eta, E \in \mathbb{R}, \eta>0$,

$$
m_{M}(z):=m_{\mu_{M}}(z)=\frac{1}{N} \operatorname{Tr} G_{M}(z)
$$

for $G_{M}$ the resolvant matrix of $M$ :

$$
G_{M}(z)=(M-z)^{-1}
$$

For $X$ an $L^{1}$ random variable,

$$
\stackrel{\circ}{X}:=X-\mathbb{E} X
$$

Note that for any $X, Y \in L^{2}$,

$$
\mathbb{E}[X Y]=\mathbb{E} X \mathbb{E} Y+\mathbb{E}[\stackrel{\circ}{X} Y] .
$$

For $f$ a function of a real variable and $\ell \geq 0, f^{(\ell)}$ denotes the $\ell$ th derivative of $f$. For $E \in \mathbb{R}$ and $\delta>0,[E \pm \delta]$ denotes the interval $[E-\delta, E+\delta]$.

For $X=X_{N}$ and $Y=Y_{N}, X \ll Y$ means that $X / Y \longrightarrow 0$ as $N \rightarrow \infty$. 


\section{Main results.}

1.1. Local law for the single ring theorem. Let $A$ be an $N \times N$ matrix (depending implicitly on $N$ ) of the form $A=U T V$, with $U, V$ some independent Haar-distributed unitary matrices and $T$ a deterministic matrix.

We make the following hypothesis.

Hypothesis 1.1. (i) There is $K>0$ independent of $N$ such that $\|T\| \leq K$.

(ii) There is $v$ a probability measure, $\eta_{0}>0$ and $C_{0}$ independent of $N$ such that

$$
\eta=\operatorname{Im} z>\eta_{0} \quad \Longrightarrow \quad\left|m_{v_{T}-v}(z)\right| \leq C_{0} N^{-1},
$$

where $m$ is the Stieltjes' transform defined at (11) and (15). $N^{-c}$.

(iii) There are $C, c>0$ independent of $N$ such that $\operatorname{Im} m_{v_{T}}(z) \leq C$ when $\operatorname{Im} z>$

Then we know, by $[22,31],{ }^{2}$ that $\mu_{A}$ converges in probability to a law $\mu$ with density $\rho$ given by (2) and support $S=\{z \in \mathbb{C} ; a \leq|z| \leq b\}$ given by (1).

Here is our main result. It will be proved in Section 4 as a consequence of the local law for the singular values proved in Theorem 1.7, which is in turn proved using the matrix subordination result in Theorem 1.5 and its consequence in Proposition 2.1.

THEOREM 1.2 (Local Single Ring Theorem). Fix $z_{0}$ such that $a<\left|z_{0}\right|<b$, $\alpha \in(0,1 / 4)$ and define $\varepsilon_{N}:=(\log N)^{-\alpha}$. Then for $f \in \mathcal{C}_{c}^{2}(\mathbb{C})$ and

$$
F_{z_{0}, \varepsilon_{N}}: \lambda \longmapsto \varepsilon_{N}^{-2} f\left(\frac{\lambda-z_{0}}{\varepsilon_{N}}\right),
$$

we have the convergence in probability, as $N \rightarrow \infty$,

$$
\int F_{z_{0}, \varepsilon_{N}}(\lambda) \mathrm{d} \mu_{A}(\lambda)-\int F_{z_{0}, \varepsilon_{N}}(\lambda) \mathrm{d} \mu(\lambda) \longrightarrow 0,
$$

where $\mu$ is the limit spectral law of A, introduced above.

REMARK 1.3. Why do we call it a local law? The convergence of $\mu_{A}$ towards $\mu$ can be considered as local with scale $\varepsilon_{N}$ at $z_{0}$ when for any test function $f$,

$$
\int f\left(\frac{\lambda-z_{0}}{\varepsilon_{N}}\right) \mathrm{d} \mu_{A}(\lambda)-\int f\left(\frac{\lambda-z_{0}}{\varepsilon_{N}}\right) \mathrm{d} \mu(\lambda) \ll \int f\left(\frac{\lambda-z_{0}}{\varepsilon_{N}}\right) \mathrm{d} \mu(\lambda) .
$$

As for a test function $f$ with enough decay at infinity, the RHT of (19) should have order at most $\mu\left(B\left(z_{0}, \varepsilon_{N}\right)\right) \approx \varepsilon_{N}^{2}$, this rewrites

$$
\int f\left(\frac{\lambda-z_{0}}{\varepsilon_{N}}\right) \mathrm{d} \mu_{A}(\lambda)-\int f\left(\frac{\lambda-z_{0}}{\varepsilon_{N}}\right) \mathrm{d} \mu(\lambda) \ll \varepsilon_{N}^{2},
$$

which is precisely the contents of the theorem.

\footnotetext{
${ }^{2}$ For the single ring theorem to hold, these hypotheses can even be weakened, as proved by Basak and Dembo in [4].
} 
REMARK 1.4. Note that we focus on the interior of the support $S$ [it is necessary at (46)]. It has been proved in $[11,23]$ that there is no eigenvalue at a macroscopic distance of $S$, but the case of the border of $S$ (i.e., $\left|z_{0}\right|=a$ or $b$ ) is not treated here.

1.2. Matrix subordination. In order to prove this local law, we need to prove a matrix subordination result, as called by Kargin in [28], where he introduced this idea for Hermitian matrices. Let $A, B$ be deterministic, depending implicitly on $N, N \times N$ matrices such that there is $K$ independent of $N$ such that

$$
\|A\|,\|B\| \leq K .
$$

Let $U, V$ be some independent $N \times N$ Haar-distributed unitary matrices and

$$
\widetilde{B}:=U B V^{*} \text {. }
$$

We introduce the matrices:

$$
\begin{aligned}
\mathbf{A} & :=\left(\begin{array}{cc}
0 & A \\
A^{*} & 0
\end{array}\right) \\
\mathbf{W}:=\left(\begin{array}{cc}
U & 0 \\
0 & V
\end{array}\right), & \mathbf{B}:=\left(\begin{array}{cc}
0 & B \\
B^{*} & 0
\end{array}\right), \\
\mathbf{H} & :=\left(\begin{array}{cc}
0 & \mathbf{\mathbf { B }}=\mathbf{W B W}^{*}, \\
(A+\widetilde{B})^{*} & A+\widetilde{B} \\
0
\end{array}\right)=\mathbf{A}+\widetilde{\mathbf{B}} .
\end{aligned}
$$

Note that the matrices $\mathbf{A}, \mathbf{B}$ and $\mathbf{H}$ have eigenvalues the singular values of respectively $A, B$ and $A+\widetilde{B}$ (and their opposites).

THEOREM 1.5. There are some complex valued functions $S_{A}(z), S_{B}(z)$ of $z=E+\mathrm{i} \eta \in \mathbb{C}^{+}$and some matrices $R_{A}(z)$ and $R_{B}(z)$ such that we have

$$
\begin{aligned}
& \mathbb{E} G_{\mathbf{H}}(z)=G_{\mathbf{A}}\left(z+S_{B}(z)\right)+R_{A}(z), \\
& \mathbb{E} G_{\mathbf{H}}(z)=G_{\mathbf{B}}\left(z+S_{A}(z)\right)+R_{B}(z),
\end{aligned}
$$

such that the functions $S_{A}(z), S_{B}(z)$, whose formulas are given at (67), satisfy

$$
N \eta^{5} \geq C \quad \Longrightarrow \quad \operatorname{Im} S_{A}(z), \operatorname{Im} S_{B}(z) \geq-\frac{C}{N \eta^{7}}
$$

and such that the matrices $R_{A}(z), R_{B}(z)$, whose formulas are given at (68), satisfy

$$
N \eta^{8} \geq C \quad \Longrightarrow \quad\left\|R_{A}(z)\right\|,\left\|R_{B}(z)\right\| \leq \frac{C}{N \eta^{6}}
$$

for a constant $C$ depending only on the $K$ of (20).

REMARK 1.6. Theorem 1.5, which will be proved in Section 5, has to be compared with Theorem A.10 and Remark A.11 of the Appendix, which give the definition of the free convolution $\boxplus$ in terms of subordination of Stieltjes' transforms and interpret subordination as a regularity criterion. 
1.3. Local laws for the singular values of $A+B$ and singular vectors delocalization. On the way to deduce Theorem 1.2 from Theorem 1.5, we will prove a key result, Proposition 2.1. Then, for almost free, we get the two following results (Theorems 1.10 and 1.12).

Let us suppose that besides the hypothesis $\|A\|,\|B\| \leq K$, there are some probability measures $v_{\mathbf{a}} \neq \delta_{0}$ and $v_{\mathbf{b}} \neq \delta_{0}$ such that as $N \rightarrow \infty$,

$$
v_{A} \longrightarrow v_{\mathbf{a}} ; \quad v_{B} \longrightarrow v_{\mathbf{b}}
$$

It is well known $[10,24]$ that we then have the convergence in probability

$$
v_{A+\widetilde{B}} \longrightarrow v
$$

with $v$ the probability measure on $\mathbb{R}_{+}$whose symmetrization $v^{s}$ [see (10)] satisfies

$$
v^{s}=v_{\mathbf{a}}^{s} \boxplus v_{\mathbf{b}}^{s} .
$$

The two next theorems give conditions for the convergence of (29) to hold at local levels.

THEOREM 1.7 (Local law 1 for the singular values of $A+\widetilde{B}$ ). Suppose that there are $\eta_{0}, C_{0}$ independent of $N$ such that

$$
\eta \geq \eta_{0} \quad \Longrightarrow \quad\left|m_{v_{A}-v_{\mathbf{a}}}(z)\right|+\left|m_{v_{B}-v_{\mathbf{b}}}(z)\right| \leq C_{0} N^{-1} .
$$

Let $p \geq 0$ and let $\phi_{N}$ be a sequence of smooth functions. Then there are $C, c>$ 0 depending only on $K, v_{\mathbf{a}}, \nu_{\mathbf{b}}, C_{0}, \eta_{0}, p$ such that with probability at least 1 $C \mathrm{e}^{-N^{c}}$,

$$
\left|\left(v_{A+\widetilde{B}}^{s}-v_{\mathbf{a}}^{s} \boxplus v_{\mathbf{b}}^{s}\right)\left(\phi_{N}\right)\right| \leq \frac{C\left\|\phi_{N}^{(p+1)}\right\|_{\infty}}{(\log N)^{p / 2}} .
$$
$\mathbb{C}^{+}$,

We define, for $\mu, \nu$, compactly supported probability measures on $\mathbb{R}$ and $z \in$

$$
\begin{aligned}
\kappa_{\mu, v}(z):=\{ & \left.m_{\mu}^{\prime}\left(z+S_{v}(z)\right)+m_{\nu}^{\prime}\left(z+S_{\mu}(z)\right)\right\}\left(z+S_{\mu}(z)+S_{v}(z)\right)^{-2} \\
& -m_{\mu}^{\prime}\left(z+S_{\nu}(z)\right) m_{\nu}^{\prime}\left(z+S_{\mu}(z)\right),
\end{aligned}
$$

where the functions $S_{\mu}, S_{\nu}$ are the subordination functions introduced in Theorem A.10.

We use the definition introduced by Kargin in [28].

DEFINITION 1.8. We say that the pair $(\mu, v)$ of probability measures on $\mathbb{R}$ is well behaved at $E \in \mathbb{R}$ if:

(a) the subordination functions $S_{\mu}, S_{v}$ have finite limits $^{3}$ with positive imaginary parts at $E$,

\footnotetext{
${ }^{3}$ It has been proved in [6] that for $\mu, v$ compactly supported, $S_{\mu}, S_{v}$ extend continuously to the whole real line, with values in $\mathbb{C}^{+} \cup \mathbb{R} \cup\{\infty\}$.
} 
(b) the value of the analytic continuation ${ }^{4}$ of the function $\kappa_{\mu, v}(z)$ at $E$ is nonzero.

REMARK 1.9. Sufficient conditions have been given, for example, by Belinschi in [5], for (a) of the previous definition to occur. As far as (b) is concerned, Kargin gave sufficient conditions in [27]. Besides, if (a) is satisfied, by the analytic continuation principle and an analysis of the function $\kappa_{\mu, v}(z)$ at infinity, we see that the set of $E$ 's where (a) holds and not (b) is discrete.

THEOREM 1.10 (Local law 2 for the singular values of $A+\widetilde{B}$ ). Suppose that the pair $\left(v_{\mathbf{a}}^{s}, v_{\mathbf{b}}^{s}\right)$ is well behaved at $E \in \mathbb{R}$ and that there is $\eta_{0}=\eta_{0}(N)$ such that

$$
\sup _{\eta \geq \eta_{0}} \eta^{2}\left|m_{v_{A}-v_{\mathbf{a}}}(z)\right|+\eta^{2}\left|m_{v_{B}-v_{\mathbf{b}}}\right| \ll N^{-1 / 4} \text {. }
$$

Then we have

$$
\max \left\{\eta_{0}, N^{-1 / 8}\right\} \ll \eta \ll 1 \Longrightarrow \frac{v_{A+\widetilde{B}}([E \pm \eta])}{2 \eta} \longrightarrow \rho(E)
$$

for the convergence in probability, where $\rho$ is the density, ${ }^{5}$ at $E$, of the limit of $v_{A+\widetilde{B}}$, that is, of $v_{\mathbf{a}}^{s} \boxplus v_{\mathbf{b}}^{s}$.

REMARK 1.11. The statement of Theorem 1.10 is close, in nature, from the one of Theorem 1.7. However, the statement of Theorem 1.10 (local law at scale $N^{-1 / 8}$ ) is stronger than the one of Theorem 1.7 (local law at logarithmic scale), but relies on stronger hypotheses [we need to know that $\left(v_{\mathbf{a}}^{s}, v_{\mathbf{b}}^{S}\right)$ is well behaved at $E \in \mathbb{R}$, which is usually hard to prove, given how little explicit formulas for $\boxplus$ are]. This dichotomy is reflected in an essential difference in their proofs: the proof of Theorem 1.10 relies on Erdôs, Schlein and Yau's method via the approximation of the Stieltjes transform of $v_{\mathbf{a}}^{s} \boxplus v_{\mathbf{b}}^{s}$ by the one of $v_{A+\widetilde{B}}$ at distance $\eta$ from the real line (see Theorem A.17), whereas the proof of Theorem 1.7 relies on Hadamard's three circles theorem and the approximation of the Stieltjes' transform of $\nu_{\mathbf{a}}^{s} \boxplus \nu_{\mathbf{b}}^{s}$ by the one of $v_{A+\widetilde{B}}$ at macroscopic distance from the real line (see Corollary A.21).

Let us now state a result about the delocalization of the singular vectors of $A+\widetilde{B}$ which will also come for free once Theorem 1.5 and Proposition 2.1 are proved.

Let $s_{a}(a=1, \ldots, N)$ denote the singular values of $A+\widetilde{B}$ and let $u_{a}(a=$ $1, \ldots, N), v_{a}(a=1, \ldots, N)$ denote some orthonormal bases such that for each $a,\left(u_{a}, v_{a}\right)$ is a pair of singular vectors for $A+\widetilde{B}$ associated to $s_{a}$ (i.e., $A+\widetilde{B}=$ $\left.\sum_{a} s_{a} u_{a} v_{a}^{*}\right)$. For each $a, i, u_{a}(i), v_{a}(i)$ denote the $i$ th components of $u_{a}, v_{a}$.

\footnotetext{
${ }^{4}$ It has been proved at Theorem 3.3 of [5] that (a) implies that the functions $S_{\mu}$ and $S_{v}$ have analytic continuations to a neighborhood of $E$, which implies that $\kappa_{\mu, v}(z)$ does so.

${ }^{5}$ It follows from Theorem 7.4 of [13] and Theorem 4.1 page 146 of [5] that there is an open set $U \subset$ $\mathbb{R}^{+}$and an analytic positive function $\rho$ on $U$ such that the limit $v$ of $v_{A+\widetilde{B}}$ is $\alpha \delta_{0}+\mathbb{1}_{U}(x) \rho(x) \mathrm{d} x$ for $\alpha:=\left(\left(v_{\mathbf{a}}+v_{\mathbf{b}}\right)(\{0\})-1\right)_{+}$.
} 
THEOREM 1.12 (Singular vectors delocalization for $A+\widetilde{B}$ ). If the pair $\left(v_{\mathbf{a}}^{S}, v_{\mathbf{b}}^{S}\right)$ is well behaved at each point in $[E-\varepsilon, E+\varepsilon](E \in \mathbb{R}, \varepsilon>0)$ and the hypotheses of Theorem 1.10 hold, then we have

$$
\begin{aligned}
& \mathbb{P}\left(\exists a , i ; | \lambda _ { a } - E | \leq \varepsilon \text { and } \left(\left|u_{a}(i)\right|^{2}>C N^{-1 / 8} \log (N)\right.\right. \\
& \text { or } \left.\left.\left|v_{a}(i)\right|^{2}>C N^{-1 / 8} \log (N)\right)\right) \\
& \quad \leq \mathrm{e}^{-c \sqrt{N}},
\end{aligned}
$$

for some constants $c, C$ depending only on the parameters of the hypotheses.

Note about the constants $c, C$ : In the proof of the local single ring theorem (Theorem 1.2) $c, C$ will denote some respectively small and large constants that might change from line to line and that depend only on the constant parameters introduced in the statement of Theorem 1.2 and in Hypothesis 1.1. In the same way, in the proofs of the matrix subordination result (Theorem 1.5), the local law for singular values and the singular vectors delocalization (Theorems 1.10 and 1.12), as well as Proposition 2.1, $c, C$ might change from line to line and depend only on the parameters introduced in the hypotheses.

2. Statement and proof of Proposition 2.1. For $\mu, v$, compactly supported probability measures on $\mathbb{R}$ and $z \in \mathbb{C}^{+}$, besides the number $\kappa_{\mu, \nu}(z)$ defined at (31), when $\kappa_{\mu, v}(z) \neq 0$, we define the numbers:

$$
\begin{aligned}
& \alpha_{\mu, v}(z):=\frac{\left|z+S_{\mu}(z)+S_{v}(z)\right|^{-2}+\left|m_{\mu}^{\prime}\left(z+S_{v}(z)\right)\right|+\left|m_{v}^{\prime}\left(z+S_{\mu}(z)\right)\right|}{\left|\kappa_{\mu, v}(z)\right|} \\
& \beta_{\mu, v}(z):=\left|z+S_{\mu}(z)+S_{v}(z)\right|^{-3}+\left|m_{\mu}^{\prime \prime}\left(z+S_{v}(z)\right)\right|+\left|m_{\nu}^{\prime \prime}\left(z+S_{\mu}(z)\right)\right|
\end{aligned}
$$

where the functions $S_{\mu}, S_{v}$ are the subordination functions introduced in Theorem A.10.

The following consequence of Theorem 1.5 will be a key result in the proof of the local version of the single ring theorem. Kargin stated very similar results in $[27,28]$ but to prove the local single ring theorem, we need to give more accurate upper bounds than the ones given in Kargin's works. We sill use this proposition for small vaues of $\eta$ in the proofs of Theorems 1.10 and 1.12 and macroscopic, as large as needed, values of $\eta$ in the proof of Theorem 1.7, which is a key step in the proof of the local single ring theorem.

Proposition 2.1. Let $s \in(0, c)$ and $z=E+\mathrm{i} \eta \in \mathbb{C}^{+}$be such that

$$
\begin{aligned}
\kappa_{v_{\mathbf{a}}^{s}, v_{\mathbf{b}}^{s}}(z) \neq 0 ; \quad N \eta^{8} \geq c ; \quad N \eta^{6} \geq \frac{c}{\alpha_{v_{\mathbf{a}}^{s}, v_{\mathbf{b}}^{s}}(z) \beta_{v_{\mathbf{a}}^{s}, v_{\mathbf{b}}^{s}}(z)}, \\
\forall z^{\prime} \in \mathbb{C}, \quad \operatorname{Im} z^{\prime} \geq \eta \quad \Longrightarrow \quad\left|m_{v_{\mathbf{a}}}\left(z^{\prime}\right)-m_{v_{A}}\left(z^{\prime}\right)\right| \leq s,
\end{aligned}
$$


then the following inequalities hold:

$$
\begin{aligned}
\left|S_{A}(z)-S_{v_{\mathbf{a}}^{s}}(z)\right| & \leq c \alpha_{v_{\mathbf{a}}^{s}, \nu_{\mathbf{b}}^{s}}(z)\left(\left(N \eta^{6}\right)^{-1}+s\right), \\
\left|S_{B}(z)-S_{\nu_{\mathbf{b}}^{s}}(z)\right| & \leq c \alpha_{\nu_{\mathbf{a}}^{s}, \nu_{\mathbf{b}}^{s}}(z)\left(\left(N \eta^{6}\right)^{-1}+s\right), \\
\left|\mathbb{E} m_{\mathbf{H}}(z)-m_{v_{\mathbf{a}}^{s} \boxplus \nu_{\mathbf{b}}^{s}}(z)\right| & \leq \frac{c \alpha_{\nu_{\mathbf{a}}^{s}, \nu_{\mathbf{b}}^{s}}(z)\left(\left(N \eta^{6}\right)^{-1}+s\right)}{\left|z+S_{\nu_{\mathbf{a}}^{s}}(z)+S_{v_{\mathbf{b}}^{s}}(z)\right|^{2}} .
\end{aligned}
$$

Proof. Note first that, by Theorem 1.5 (whose proof is postponed to Section 5),

$$
\mathbb{E} m_{\mathbf{H}}(z)=\frac{1}{2 N} \operatorname{Tr}\left(\mathbb{E} G_{\mathbf{H}}(z)\right)=-\frac{1}{z+S_{A}(z)+S_{B}(z)} .
$$

Indeed, with our definition of (67) [and its analogue for $S_{A}(z)$ ], we have

$$
\begin{aligned}
z+S_{A}(z)+S_{B}(z) & =\frac{\mathbb{E}\left[z m_{\mathbf{H}}(z)-f_{B}(z)-f_{A}(z)\right]}{\mathbb{E}\left[m_{\mathbf{H}}(z)\right]} \\
& =\frac{\mathbb{E}\left[\frac{1}{2 N} \operatorname{Tr}\left((z-\mathbf{A}-\mathbf{W B W})\left(\mathbf{A}+\mathbf{W B W}^{*}-z\right)^{-1}\right)\right]}{\mathbb{E}\left[m_{\mathbf{H}}(z)\right]} \\
& =-\frac{1}{\mathbb{E}\left[m_{\mathbf{H}}(z)\right]} .
\end{aligned}
$$

Besides,

$$
m_{v_{\mathbf{a}}^{s} \boxplus v_{\mathbf{b}}^{s}}(z)=-\frac{1}{z+S_{v_{\mathbf{a}}^{s}}(z)+S_{v_{\mathbf{b}}^{s}}(z)},
$$

hence

$$
\mathbb{E} m_{\mathbf{H}}(z)-m_{v_{\mathbf{a}}^{s} \boxplus v_{\mathbf{b}}^{s}}(z)=\frac{S_{\nu_{\mathbf{a}}^{s}}(z)+S_{\nu_{\mathbf{b}}^{s}}(z)-S_{A}(z)-S_{B}(z)}{\left(z+S_{A}(z)+S_{B}(z)\right)\left(z+S_{v_{\mathbf{a}}^{s}}(z)+S_{v_{\mathbf{b}}^{s}}(z)\right)},
$$

and the third equation of the lemma follows from its two first ones. Let us prove them.

For $z \in \mathbb{C}^{+}$, we define the set

$$
O_{z}:=\left\{\left(s_{1}, s_{2}\right) \in \mathbb{C} ; s_{1}+s_{2} \neq-z, z+s_{1}, z+s_{2} \in \mathbb{C}^{+}\right\}
$$

and for $\mu, v$ probability measures on $\mathbb{R}$, we define the function $F_{\mu, v, z}: O_{z} \rightarrow \mathbb{C}^{2}$ by

$$
F_{\mu, v, z}\left(\begin{array}{l}
s_{1} \\
s_{2}
\end{array}\right):=\left(\begin{array}{l}
m_{\mu^{s}}\left(z+s_{2}\right)+\left(z+s_{1}+s_{2}\right)^{-1} \\
m_{v^{s}}\left(z+s_{2}\right)+\left(z+s_{1}+s_{2}\right)^{-1}
\end{array}\right) .
$$

With the notations of Theorem A.10, we have

$$
F_{\mu, v, z}\left(\begin{array}{c}
S_{\mu^{s}}(z) \\
S_{v^{s}}(z)
\end{array}\right)=\left(\begin{array}{l}
0 \\
0
\end{array}\right) .
$$


We shall apply it for $\mu=v_{\mathbf{a}}$ and $v=v_{\mathbf{b}}$, yielding

$$
F_{v_{\mathbf{a}}, \nu_{\mathbf{b}}, z}\left(\begin{array}{l}
S_{v_{\mathbf{a}}^{s}}(z) \\
S_{v_{\mathbf{b}}^{s}}(z)
\end{array}\right)=\left(\begin{array}{l}
0 \\
0
\end{array}\right)
$$

A similar system can be written for $S_{A}$ and $S_{B}$ : by (37), (24) and (25)

$$
\begin{aligned}
& m_{\mathbf{A}}\left(z+S_{B}(z)\right)+\frac{1}{z+S_{A}(z)+S_{B}(z)}=-\frac{1}{2 N} \operatorname{Tr} R_{A}(z)=: r_{A}(z), \\
& m_{\mathbf{B}}\left(z+S_{A}(z)\right)+\frac{1}{z+S_{A}(z)+S_{B}(z)}=-\frac{1}{N} \operatorname{Tr} R_{B}(z)=: r_{B}(z),
\end{aligned}
$$

so that

$$
F_{v_{A}, v_{B}, z}\left(\begin{array}{l}
S_{A}(z) \\
S_{B}(z)
\end{array}\right)=\left(\begin{array}{c}
r_{A}(z) \\
r_{B}(z)
\end{array}\right)
$$

and that by hypothesis,

$$
\begin{aligned}
& F_{v_{\mathbf{a}}, v_{\mathbf{b}}, z}\left(\begin{array}{c}
S_{A}(z) \\
S_{B}(z)
\end{array}\right)=\left(\begin{array}{c}
\hat{r}_{A}(z) \\
\hat{r}_{B}(z)
\end{array}\right) \\
& \text { with }\left|\hat{r}_{A}(z)-r_{A}(z)\right|+\left|\hat{r}_{B}(z)-r_{B}(z)\right| \leq s .
\end{aligned}
$$

Let us now consider the intermediate system:

$$
F_{v_{\mathbf{a}}, \nu_{\mathbf{b}}, z}\left(\begin{array}{c}
\tilde{S}_{A}(z) \\
\tilde{S}_{B}(z)
\end{array}\right)=\left(\begin{array}{c}
r_{A}(z) \\
r_{B}(z)
\end{array}\right) .
$$

First, we shall upper-bound the distance between the solution $\left(S_{\nu_{\mathbf{a}}^{s}}(z), S_{\nu_{\mathbf{b}}^{s}}(z)\right)$ of (39) and the solution $\left(\tilde{S}_{A}(z), \tilde{S}_{B}(z)\right)$ of (44) [using Kantorovich's Theorem A.15 and the fact that the derivative of $F_{v_{A}, v_{B}, z}$ is not too small and that $r_{A}(z), r_{B}(z)$ are small]. Second, we shall upper-bound the distance between $\left(\tilde{S}_{A}(z), \tilde{S}_{B}(z)\right)$ [as a solution of (44) again] and the solution $\left(S_{A}(z), S_{B}(z)\right)$ of (43) (using the same ideas).

Let us upper-bound the distance between the solutions of (39) and (44) thanks to Kantorovich's Theorem A.15. For

$$
S_{z}:=\left(\begin{array}{c}
S_{v_{\mathbf{a}}^{s}}(z) \\
S_{v_{\mathbf{b}}^{s}}(z)
\end{array}\right) ; \quad M_{z}:=\left\|\left(F_{v_{\mathbf{a}}, v_{\mathbf{b}}, z}^{\prime}\left(S_{z}\right)\right)^{-1}\right\|,
$$

by Theorem A.15, we have

$$
\left|S_{A}(z)-S_{v_{\mathbf{a}}^{s}}(z)\right|+\left|S_{B}(z)-S_{\nu_{\mathbf{b}}^{s}}(z)\right| \leq 100 M_{z}\left(\left|r_{A}(z)\right|+\left|r_{B}(z)\right|\right)
$$

as soon as

$$
100 M_{z}^{2}\left(\left|r_{A}(z)\right|+\left|r_{B}(z)\right|\right)\left\|F_{v_{A}, v_{B}, z}^{\prime \prime}\left(S_{z}\right)\right\|<1
$$


(the 100 is here to avoid any norm choice issue, as Theorem A.15 is stated for the Euclidean norm). The derivative

$$
\begin{aligned}
& F_{v_{\mathbf{a}}, v_{\mathbf{b}}, z}^{\prime}\left(\begin{array}{l}
s_{1} \\
s_{2}
\end{array}\right) \\
& \quad=\left(\begin{array}{cc}
-\left(z+s_{1}+s_{2}\right)^{-2} & -\left(z+s_{1}+s_{2}\right)^{-2}+m_{v_{\mathbf{a}}^{s}}^{\prime}\left(z+s_{2}\right) \\
-\left(z+s_{1}+s_{2}\right)^{-2}+m_{v_{\mathbf{b}}^{s}}^{\prime}\left(z+s_{1}\right) & -\left(z+s_{1}+s_{2}\right)^{-2}
\end{array}\right)
\end{aligned}
$$

has determinant

$$
\begin{aligned}
\operatorname{det} F_{v_{\mathbf{a}}, \nu_{\mathbf{b}}, z}^{\prime}\left(\begin{array}{l}
s_{1} \\
s_{2}
\end{array}\right) \\
\quad=\left\{m_{v_{\mathbf{a}}^{s}}^{\prime}\left(z+s_{2}\right)+m_{\nu_{\mathbf{b}}^{s}}^{\prime}\left(z+s_{1}\right)\right\}\left(z+s_{1}+s_{2}\right)^{-2}-m_{v_{\mathbf{a}}^{s}}^{\prime}\left(z+s_{2}\right) m_{v_{\mathbf{b}}^{s}}^{\prime}\left(z+s_{1}\right),
\end{aligned}
$$

so that

$$
M_{z} \leq \alpha_{v_{\mathbf{a}}^{s}, v_{\mathbf{b}}^{s}}(z)
$$

Its second derivative is bounded by

$$
100 \beta_{v_{\mathbf{a}}^{s}, \nu_{\mathbf{b}}^{s}}(z) \text {. }
$$

This proves that under the hypotheses of the lemma, the distance between the solutions of (39) and (44), that is, between $\left(\begin{array}{c}S_{v_{\mathbf{a}}^{s}}(z) \\ S_{v_{\mathbf{b}}}(z)\end{array}\right)$ and $\left(\begin{array}{c}\tilde{S}_{A}(z) \\ \tilde{S}_{B}(z)\end{array}\right)$, is upper-bounded by the first part of the common right-hand side of (34) and (35).

Upper-bounding the distance between the solutions of (44) and (43) goes along the same lines, and gives the second part (the one involving $s$ ) of the common right-hand side of (34) and (35).

\section{Proofs of Theorems 1.7, 1.10 and 1.12 (Local laws and singular vectors delocalization for $A+\widetilde{B}$ ).}

\subsection{Proof of Theorem 1.7 (Local law 1).}

LEMMA 3.1. Let $\mathcal{K}$ be a fixed compact subset of $\mathbb{C}^{+}$. Then there is $C=$ $C(\mathcal{K})>0$ such that for any $\delta>0$,

$$
\mathbb{P}\left(\sup _{z \in \mathcal{K}}\left|m_{\mathbf{H}}(z)-\mathbb{E} m_{\mathbf{H}}(z)\right|>\delta\right) \leq C \mathrm{e}^{-C \delta^{2} N^{2}} .
$$

PROOF. The lemma can be proved as Corollary 6 of [26].

Let us now prove Theorem 1.7. By Lemma A.13, Proposition 2.1, Lemma 3.1 and Corollary A.21, we know that there are $C, c>0$ such that we have, with probability at least $1-C \mathrm{e}^{-N^{c}}$,

$$
\sup \left\{\left|m_{v_{A+\widetilde{B}}^{s}-v_{\mathbf{a}}^{s} \boxplus \nu_{\mathbf{b}}^{s}}(z)\right| ; z=E+\mathrm{i} \eta,|E| \leq 3 K, \eta=\frac{C}{\sqrt{\log N}}\right\} \leq C \mathrm{e}^{-c \sqrt{\log N}} .
$$

By Corollary A.19, we conclude. 
3.2. Proof of Theorem 1.10 (Local law 2). The proof is a direct application of Lemma 3.1 and Proposition 2.1 and of Erdős, Schlein and Yau's method (see Theorem A.17 in the Appendix).

3.3. Proof of Theorem 1.12 (Singular vectors delocalization). The proof is a copy of the one of Theorem 4 in [27]. Let us give the main lines. First, we note that for any $a$, as $\left(u_{a}, v_{a}\right)$ of unit singular vectors associated to the singular value $s_{a}$ of $A+\widetilde{B}$, the vector

$$
w_{a, \pm}:=\frac{1}{\sqrt{2}}\left(u_{a} \pm v_{a}\right)
$$

is an eigenvector of $\mathbf{H}$ associated to the eigenvalue $\pm s_{a}$.

Then we use the classical trick by Erdős, Schlein and Yau that for $E= \pm s_{a}$,

$$
\left|w_{a, \pm}(i)\right|^{2} \leq \eta\left|G_{\mathbf{H}}(E+\mathrm{i} \eta)\right|_{i i} .
$$

Then we prove that if $1 \gg \eta \gg \max \left\{\eta_{0}, N^{-1 / 8}\right\}$, then

$$
\left\|\mathbb{E} G_{\mathbf{H}}(x+\mathrm{i} \eta)\right\|=O(1),
$$

uniformly on $E$ on $x \in[E \pm \varepsilon]$, so that

$$
\left|\mathbb{E} G_{\mathbf{H}}(E+\mathrm{i} \eta)_{i i}\right|=O(1) .
$$

Then some concentration estimates allow to conclude.

4. Proof of Theorem 1.2 (Local single ring theorem). It is well known (see, e.g., [15], Section 4) that for any $A \in \mathcal{M}_{N}(\mathbb{C})$ and any $F \in \mathcal{C}_{c}^{2}(\mathbb{C})$,

$$
\int F(\lambda) \mathrm{d} \mu_{A}(\lambda)=\frac{1}{2 \pi} \int_{z \in \mathbb{C}} \Delta F(z)\left(\int \log |s| \mathrm{d} \nu_{z-A}(s)\right) \mathrm{d} \operatorname{Re}(z) \mathrm{d} \operatorname{Im}(z) .
$$

Here, we get

$$
\begin{aligned}
& \int F_{z_{0}, \varepsilon_{N}}(\lambda) \mathrm{d} \mu_{A}(\lambda) \\
& =\frac{1}{N} \sum_{i=1}^{N} F_{z_{0}, \varepsilon_{N}}\left(\lambda_{i}\right) \\
& =\frac{1}{2 \pi} \int\left(\Delta F_{z_{0}, \varepsilon_{N}}\right)(z) \int \log |s| \mathrm{d} v_{z-A}(s) \mathrm{d} \operatorname{Re}(z) \mathrm{d} \operatorname{Im}(z) \\
& =\frac{1}{2 \pi \varepsilon_{N}^{4}} \int(\Delta f)\left(\frac{z-z_{0}}{\varepsilon_{N}}\right) \int \log |s| \mathrm{d} v_{z-A}(s) \mathrm{d} \operatorname{Re}(z) \mathrm{d} \operatorname{Im}(z) \\
& =\frac{1}{2 \pi \varepsilon_{N}^{2}} \int \Delta f\left(z^{\prime}\right) \int \log |s| \mathrm{d} v_{z_{0}+\varepsilon_{N} z^{\prime}-A}(s) \mathrm{d} \operatorname{Re}\left(z^{\prime}\right) \mathrm{d} \operatorname{Im}\left(z^{\prime}\right) .
\end{aligned}
$$


In the same way, we have [using an integration by parts at (46) because $z_{0}$ has been chosen in the interior of the support of the function $\rho$ of (2)],

$$
\begin{aligned}
& \int F_{z_{0}, \varepsilon_{N}}(\lambda) \mathrm{d} \mu(\lambda) \\
& \quad=\frac{1}{\varepsilon_{N}^{2}} \int f\left(\frac{z-z_{0}}{\varepsilon_{N}}\right) \rho(z) \mathrm{d} \operatorname{Re}(z) \mathrm{d} \operatorname{Im}(z) \\
& =\frac{1}{2 \pi \varepsilon_{N}^{2}} \int f\left(\frac{z-z_{0}}{\varepsilon_{N}}\right) \Delta_{z}\left(\int \log |x| \nu_{\infty, z}(\mathrm{~d} x)\right) \mathrm{d} \operatorname{Re}(z) \mathrm{d} \operatorname{Im}(z) \\
& =\frac{1}{2 \pi \varepsilon_{N}^{4}} \int(\Delta f)\left(\frac{z-z_{0}}{\varepsilon_{N}}\right) \int \log |x| v_{\infty, z}(\mathrm{~d} x) \mathrm{d} \operatorname{Re}(z) \mathrm{d} \operatorname{Im}(z) \\
& =\frac{1}{2 \pi \varepsilon_{N}^{2}} \int(\Delta f)\left(z^{\prime}\right) \int \log |x| \nu_{\infty, z_{0}+\varepsilon_{N} z^{\prime}}(\mathrm{d} x) \mathrm{d} \operatorname{Re}\left(z^{\prime}\right) \mathrm{d} \operatorname{Im}\left(z^{\prime}\right)
\end{aligned}
$$

Hence, to prove that both expressions are equal up to an error term tending to zero in probability as $N \rightarrow \infty$, by Lemma 3.1 of [32], we need to prove that:

(i) for any $z^{\prime} \in \mathbb{C}$, we have, for the convergence in probability,

$$
\varepsilon_{N}^{-2}\left|\int \log \right| x\left|\mathrm{~d}\left(v_{z_{0}+\varepsilon_{N} z^{\prime}-A}-v_{\infty, z_{0}+\varepsilon_{N} z^{\prime}}\right)(x)\right| \underset{N \rightarrow \infty}{\longrightarrow} 0,
$$

(ii) for any $R>0$, the sequence

$$
\varepsilon_{N}^{-4} \int_{z^{\prime} \in B(0, R)} \mathbb{E}\left[\left|\int \log \right| x\left|\mathrm{~d}\left(v_{z_{0}+\varepsilon_{N} z^{\prime}-A}-v_{\infty, z_{0}+\varepsilon_{N} z^{\prime}}\right)(x)\right|^{2}\right] \mathrm{d} \operatorname{Re}\left(z^{\prime}\right) \mathrm{d} \operatorname{Im}\left(z^{\prime}\right)
$$

is bounded.

We shall in fact prove that for any $R>0$, uniformly in $z^{\prime} \in B(0, R)$,

$$
\varepsilon_{N}^{-4} \mathbb{E}\left[\left|\int \log \right| x\left|\mathrm{~d}\left(v_{z_{0}+\varepsilon_{N} z^{\prime}-A}-v_{\infty, z_{0}+\varepsilon_{N} z^{\prime}}\right)(x)\right|^{2}\right] \underset{N \rightarrow \infty}{\longrightarrow} 0,
$$

which will prove (i) and (ii) in the same time.

So let us fix $R>0$.

Let us now choose a positive integer $p$ and $\epsilon>0$ such that

$$
4 \alpha(p+2)+2 \epsilon(p+1)<p
$$

(which is possible since $4 \alpha<1$ ) and set

$$
t_{N}:=(\log N)^{-(2 \alpha+\epsilon)} \text {. }
$$

Then (47) implies that

$$
\frac{t_{N}^{-(p+1)}}{(\log N)^{p / 2}} \ll \varepsilon_{N}^{2}
$$


and as $\epsilon>0$, for any $k \geq 1$,

$$
t_{N}\left|\log t_{N}\right|^{k} \ll \varepsilon_{N}^{2}
$$

Let $\varphi_{t_{N}}$ be a smooth function with support contained in $\left[t_{N} / 2,3 K+1\right]$, taking values in $[0,1]$, equal to 1 on $\left[t_{N}, 3 K\right]$. We can construct a sequence of such functions such that for a certain constant $C$ independent of $N$, for all $\ell=0, \ldots, p+1$,

$$
\left\|\varphi_{t_{N}}^{(\ell)}\right\|_{\infty} \leq C t_{N}^{-\ell}
$$

We set

$$
\log _{\geq t_{N}}(x):=\varphi_{t_{N}}(x) \log (x) \quad \text { and } \quad \log _{<t_{N}}(x):=\left(1-\varphi_{t_{N}}(x)\right) \log (x) .
$$

Then we have, for $\xi=z_{0}+\varepsilon_{N} z^{\prime}$,

$$
\begin{aligned}
& \left|\int \log \right| x\left|\mathrm{~d}\left(v_{\xi-A}-v_{\infty, \xi}\right)(x)\right| \\
& \leq\left|\int \log _{\geq t_{N}}\right| x\left|\mathrm{~d}\left(v_{\xi-A}-v_{\infty, \xi}\right)(x)\right|+\left|\int \log _{<t_{N}}\right| x\left|\mathrm{~d} v_{\xi-A}(x)\right| \\
& \quad+\left|\int \log _{<t_{N}}\right| x\left|\mathrm{~d} v_{\infty, \xi}(x)\right| .
\end{aligned}
$$

Let us treat the three terms in the RHS of (53) separately.

- By Theorem $1.7,(51)$ and (49), with probability at least $1-C \mathrm{e}^{-N^{c}}$, uniformly in $z^{\prime} \in B(0, R)$, we have

$$
\left|\int \log _{\geq t_{N}}\right| x\left|\mathrm{~d}\left(v_{\xi-A}-v_{\infty, \xi}\right)(x)\right| \leq C \frac{t_{N}^{-(p+1)}}{(\log N)^{p / 2}} .
$$

But by (49), the RHT is $\ll \varepsilon_{N}^{2}$. As, on the complementary of the above event, we have the domination inequality

$$
\varepsilon_{N}^{-4}\left|\int \log _{\geq t_{N}}\right| x\left|\mathrm{~d}\left(v_{\xi-A}-v_{\infty, \xi}\right)(x)\right|^{2} \leq 4 \log N\left(\left|\log t_{N}\right|+K\right)^{2} \ll C \mathrm{e}^{N^{c}},
$$

we deduce that uniformly in $z^{\prime} \in B(0, R)$,

$$
\mathbb{E}\left[\varepsilon_{N}^{-4}\left|\int \log _{\geq t_{N}}\right| x\left|\mathrm{~d}\left(v_{\xi-A}-v_{\infty, \xi}\right)(x)\right|^{2}\right] \underset{N \rightarrow \infty}{\longrightarrow} 0
$$

- Let us now treat the close-to-zero terms.

LEMMA 4.1. Let $\mathcal{K}$ be a compact subset of $\mathbb{C}$ which does not contain 0 .

(a) Let $C$ be as in Hypothesis 1.1(iii). Then for any $\xi \in \mathbb{C}, t \in(0,1)$,

$$
\left|\int_{[0, t]} \log \right| x\left|\left(v_{\infty, \xi}\right)(\mathrm{d} x)\right| \leq C t(1-\log (t)) .
$$


(b) There are some constants $A_{\mathcal{K}}, a_{\mathcal{K}}>0$ such that for all $N$ large enough, for all $\xi \in \mathcal{K}$ and all $y>0$,

$$
\mathbb{E}\left[v_{\xi-A}([-y, y])\right] \leq A_{\mathcal{K}} \max \left\{y, N^{-a_{\mathcal{K}}}\right\} .
$$

(c) Let $N \geq 1$ and $v$ be a probability measure on $\mathbb{R}$ such that for some constants $A, a>0$, for all $y>0$,

$$
v([-y, y]) \leq A \max \left\{y, N^{-a}\right\} .
$$

Then there is $A^{\prime}=A^{\prime}(A, a)$ such that for any $t \in\left[N^{-a}, 1\right]$, we have

$$
\int_{\left[N^{-a}, t\right]}|\log | x||^{2} \mathrm{~d} \nu(x) \leq A^{\prime}\left(t|\log t|^{2}+N^{-a}|\log N|^{2}\right) .
$$

(d) There are some positive constants $c_{\mathcal{K}}, C_{\mathcal{K}}$ such that for $N \geq 1$ large enough, for all $\xi \in \mathcal{K}, u>0$,

$$
\mathbb{P}\left(s_{\min }(\xi-A) \leq u\right) \leq C_{\mathcal{K}} u^{c} \mathcal{K}{ }^{C_{\mathcal{K}}}
$$

and such that for any $\delta>0$, we have, for all $\xi \in \mathcal{K}$,

$$
\mathbb{E}\left[\left|\log \left(s_{\min }(\xi-A)\right)\right|^{4} \mathbb{1}_{s_{\min }(\xi-A) \leq N^{-\delta}}\right] \leq C_{\mathcal{K}} N^{C_{\mathcal{K}}-\delta c_{\mathcal{K}}}(\log N)^{4} .
$$

Proof. (a) For $C$ as in Hypothesis 1.1(iii), by Lemma A.16, we have $\operatorname{Im} m_{v} \leq$ $C$ on $\mathbb{C}^{+}$, hence $\operatorname{Im} m_{v^{s}} \leq C$ on $\mathbb{C}^{+}$. So by Lemma A.16, for any $\xi \in \mathbb{C}, v_{\infty, \xi}=$ $\nu^{s} \boxplus \delta_{|\xi|}^{s}$ has a density with respect to the Lebesgue measure, which is bounded by $C / \pi$. Thus, for $t \in(0,1)$,

$$
\begin{aligned}
\left|\int_{[0, t]} \log \right| x\left|\left(v_{\infty, \xi}\right)(\mathrm{d} x)\right| & \leq-(C / \pi) \int_{0}^{t} \log x \mathrm{~d} x \\
& =-(C / \pi) t(\log (t)-1) .
\end{aligned}
$$

(b) Follows directly from Lemmas 13 and 15 of [22] (the fact that the estimate is uniform in $\xi$ as $\xi$ stays bounded and bounded away from zero follows from a careful look at the arguments of [22]).

(c) Can be found in the proof of [22], Proposition 4(i), page 1208.

(d) The first part follows from Theorem 1.1 of [31] by Rudelson and Vershynin, as for $\xi \neq 0, s_{\min }(\xi-A)=|\xi| s_{\min }\left(U^{*} V^{*}-T / \xi\right)$. Then to compute the expectation, we will use the fact that for any positive random variable $X$, any $\alpha>0$ and any $\varepsilon \in(0,1]$,

$$
\begin{aligned}
& \mathbb{E}\left[|\log (X)|^{\alpha} \mathbb{1}_{X \leq \varepsilon}\right] \\
& \quad=\alpha \int_{0}^{\varepsilon} \mathbb{P}(X \leq u)|\log u|^{\alpha-1} \frac{\mathrm{d} u}{u}+\mathbb{P}(X \leq \varepsilon)|\log \varepsilon|^{\alpha},
\end{aligned}
$$


so that

$$
\begin{aligned}
& \mathbb{E}\left[\left|\log \left(s_{\min }(\xi-A)\right)\right|^{4} \mathbb{1}_{s_{\min }(\xi-A) \leq N^{-\delta}}\right] \\
& \quad \leq-2 C_{\mathcal{K}} N^{C_{\mathcal{K}}} \int_{0}^{N^{-\delta}} u^{c_{\mathcal{K}}-1} \log ^{3}(u) \mathrm{d} u+C_{\mathcal{K}} c_{\mathcal{K}} \delta N^{C_{\mathcal{K}}-c_{\mathcal{K}} \delta}(\log N)^{4} \\
& \quad \leq C_{\mathcal{K}}^{\prime} N^{C_{\mathcal{K}}-\delta c_{\mathcal{K}}}\left(1+(\log N)^{4}\right)
\end{aligned}
$$

We know that for $N$ large enough, for any $\xi \in B\left(z_{0}, R \varepsilon_{N}\right)$, the support of $v_{\infty, \xi}=v^{s} \boxplus \delta_{|\xi|}^{s}$ and the spectrum of $|\xi-A|$ are contained in $[-3 K, 3 K]$, so their intersection with the support of the function $\log _{<t_{N}}$ defined at (52) is contained in $\left[0, t_{N}\right]$. As the function $\varphi_{t_{N}}$ only takes values in $[0,1]$, we have

$$
\left|\int \log _{<t_{N}}\right| x\left|\mathrm{~d} v_{\infty, \xi}(x)\right| \leq-2 \int_{\left[0, t_{N}\right]} \log x \mathrm{~d} \nu_{\infty, \xi}(x)
$$

and

$$
\begin{aligned}
\left|\int \log _{<t_{N}}\right| x\left|\mathrm{~d} \nu_{\xi-A}(x)\right| \leq & -2 \int_{\left[0, t_{N}\right]} \log x \mathrm{~d} \nu_{\xi-A}(x) \\
\leq & -2 \int_{\left[0, N^{-\delta}\right]} \log x \mathrm{~d} \nu_{\xi-A}(x) \\
& -2 \int_{\left[N^{-\delta}, N^{-a} \mathcal{K}\right]} \log x \mathrm{~d} \nu_{\xi-A}(x) \\
& -2 \int_{\left[N^{-a} \mathcal{K}_{\left., t_{N}\right]}\right.} \log x \mathrm{~d} \nu_{\xi-A}(x),
\end{aligned}
$$

where $\delta>0$ is chosen such that for $c_{\mathcal{K}}, C_{\mathcal{K}}$ is in (d) of the previous lemma, we have $C_{\mathcal{K}}-\delta c_{\mathcal{K}}<0$

$\bullet$ By (55), (a) of Lemma 4.1 and (50), we know that

$$
\left|\int \log _{<t_{N}}\right| x\left|\mathrm{~d} v_{\infty, \xi}(x)\right| \ll \varepsilon_{N}^{2} .
$$

- Let us now treat the three terms of the RHS of (56).

First term of the RHS of (56): We always have

$$
\int_{\left[0, N^{-\delta}\right]}|\log | x|| \mathrm{d} \nu_{\xi-A}(x) \leq \nu_{\xi-A}\left(\left[0, N^{-\delta}\right]\right)\left|\log \left(s_{\min }(\xi-A)\right)\right| \mathbb{1}_{s_{\min }(\xi-A) \leq N^{-\delta}}
$$

Let us now take the second moment. By Cauchy-Schwarz, we have

$$
\begin{aligned}
& \mathbb{E}\left[\left(\int_{\left[0, N^{-\delta}\right]}|\log | x|| \mathrm{d} v_{\xi-A}(x)\right)^{2}\right] \\
& \quad \leq \mathbb{E}\left[\left(\nu_{\xi-A}\left(\left[0, N^{-\delta}\right]\right)\right)^{2}\left|\log \left(s_{\min }(\xi-A)\right)\right|^{2} \mathbb{1}_{\left.s_{\min }(\xi-A) \leq N^{-\delta}\right]}\right. \\
& \quad \leq \sqrt{\mathbb{E}\left[v_{\xi-A}\left(\left[0, N^{-\delta}\right]\right)^{4}\right] \mathbb{E}\left[\left|\log \left(s_{\min }(\xi-A)\right)\right|^{4} \mathbb{1}_{s_{\min }(\xi-A) \leq N^{-\delta}}\right]}
\end{aligned}
$$


Then we use (b) of Lemma 4.1 (plus the fact that $x^{4} \leq x$ when $x \in[0,1]$ ) to upper bound $\mathbb{E}\left[v_{\xi-A}\left(\left[0, N^{-\delta}\right]\right)^{4}\right]$ and (d) of Lemma 4.1 to upper bound $\mathbb{E}\left[\mid \log \left(s_{\min }(\xi-\right.\right.$ $\left.A))\left.\right|^{4} \mathbb{1}_{s_{\min }(\xi-A) \leq N^{-\delta}}\right]$. As we chose $\delta$ so that $C_{\mathcal{K}}-\delta c_{\mathcal{K}}<0$, we get that

$$
\mathbb{E}\left[\left(\int_{\left[0, N^{-\delta}\right]} \log x \mathrm{~d} \nu_{\xi-A}(x)\right)^{2}\right] \ll \varepsilon_{N}^{4} .
$$

Second term of the RHS of (56): We have

$$
\begin{aligned}
\mathbb{E}\left[\left|\int_{\left[N^{-\delta}, N^{-a} \mathcal{K}\right]} \log x \mathrm{~d} \nu_{\xi-A}(x)\right|^{2}\right] & \leq \delta \log N \mathbb{E}\left[\nu_{\xi-A}\left(\left[N^{-\delta}, N^{-a \mathcal{K}}\right]\right)\right] \\
& \leq C \delta(\log N) N^{-a} \mathcal{K} \ll \varepsilon_{N}^{4},
\end{aligned}
$$

where we used (b) of Lemma 4.1.

Third term of the RHS of (56): By (b) and (c) of Lemma 4.1 and (50) (using Cauchy-Schwarz again, as above), we can claim that

$$
\mathbb{E}\left[\left(\int_{\left[N^{-a} \mathcal{K}, t_{N}\right]} \log x \mathrm{~d} \nu_{\xi-A}(x)\right)^{2}\right] \ll \varepsilon_{N}^{4} .
$$

- Let us conclude the proof. By what precedes, we have proved that the RHS of (56) has second moment $\ll \varepsilon_{N}^{4}$, uniformly in $z^{\prime} \in B(0, R)$. Besides, by (57), we have proved that the (deterministic) RHT of (55) is $\ll \varepsilon_{N}^{2}$, uniformly in $z^{\prime} \in$ $B(0, R)$. This proves that the close-to-zero terms in (53) have second moments $\ll \varepsilon_{N}^{4}$, uniformly in $z^{\prime} \in B(0, R)$. At (54), we proved that the same holds for the far-from-zero term in (53). This completes the proof of the theorem.

5. Proof of Theorem 1.5 (Matrix subordination). This proof goes roughly along the same lines as the one of Theorem 2 of the paper [28] by Kargin. The main difficulty is to give a Schwinger-Dyson equation adapted to our context (Lemma 5.2), which forces us to introduce the linear form $\tau$ of (59) (from the point of view of quantum probability theory, which identifies the normalized trace to an expectation, $\tau$ can be assimilated to a conditional expectation).

5.1. Preliminaries. First, one can easily see, by left and right invariance of the Haar measure, that one can suppose that $A$ and $B$ are diagonal matrices with nonnegative entries, so that

$$
A^{*}=A ; \quad B^{*}=B .
$$

To state our forthcoming equation (63), we define the map:

$$
\begin{aligned}
\tau: \mathcal{M}_{2 N}(\mathbb{C}) & =\left(\begin{array}{ll}
\mathcal{M}_{N}(\mathbb{C}) & \mathcal{M}_{N}(\mathbb{C}) \\
\mathcal{M}_{N}(\mathbb{C}) & \mathcal{M}_{N}(\mathbb{C})
\end{array}\right) \longrightarrow\left(\begin{array}{cc}
\mathcal{M}_{N}(\mathbb{C}) & 0 \\
0 & \mathcal{M}_{N}(\mathbb{C})
\end{array}\right), \\
\left(\begin{array}{ll}
A & B \\
C & D
\end{array}\right) & \longmapsto\left(\begin{array}{cc}
\frac{1}{N} \operatorname{Tr} A & 0 \\
0 & \frac{1}{N} \operatorname{Tr} D
\end{array}\right)
\end{aligned}
$$


where the complex numbers $\frac{1}{N} \operatorname{Tr} A$ and $\frac{1}{N} \operatorname{Tr} D$ are assimilated to the corresponding scalar matrices.

REMARK 5.1. Let us introduce the matrix:

$$
P:=\left(\begin{array}{ll}
0 & I \\
I & 0
\end{array}\right)
$$

( $I$ denotes the identity matrix), which satisfies

$$
\forall X_{1}, X_{2}, Y_{1}, Y_{2} \in \mathcal{M}_{N}(\mathbb{C}), \quad P\left(\begin{array}{cc}
X_{1} & Y_{1} \\
Y_{2} & X_{2}
\end{array}\right) P^{-1}=\left(\begin{array}{cc}
X_{2} & Y_{2} \\
Y_{1} & X_{1}
\end{array}\right) .
$$

Thus by (58), $\mathbf{A}$ and $\mathbf{B}$ are invariant under conjugation by $P$ and the matrix $\mathbf{W}$ is invariant, in law, under conjugation by $P$. We deduce that the (random or deterministic) matrices $\mathbf{H}, G_{\mathbf{H}}(z)$ and $\mathbb{E} G_{\mathbf{H}}(z)$ are invariant, in law, under conjugation by $P$. It implies that

$$
\mathbb{E}\left[\tau\left(G_{\mathbf{H}}(z)\right)\right] ; \quad \mathbb{E}\left[\tau\left(G_{\mathbf{H}}(z) \widetilde{\mathbf{B}}\right)\right]
$$

are scalar $2 N \times 2 N$ matrices equal to respectively $\mathbb{E}\left[m_{\mathbf{H}}(z)\right] I$ and $\mathbb{E}\left[f_{B}(z)\right] I$ for

$$
f_{B}(z):=\frac{1}{2 N} \operatorname{Tr}\left(G_{\mathbf{H}}(z) \widetilde{\mathbf{B}}\right) .
$$

The following lemma is the Schwinger-Dyson equation of our problem.

LEMMA 5.2. For any $z$,

$$
\mathbb{E}\left[\tau\left(G_{\mathbf{H}}(z)\right) \widetilde{\mathbf{B}} G_{\mathbf{H}}(z)\right]=\mathbb{E}\left[\tau\left(G_{\mathbf{H}}(z) \widetilde{\mathbf{B}}\right) G_{\mathbf{H}}(z)\right] .
$$

PROOF. It suffices to prove that the element of $\mathcal{M}_{2 N}(\mathbb{C}) \otimes \mathcal{M}_{2 N}(\mathbb{C})$

$$
\mathbb{E}\left[G_{\mathbf{H}}(z) \otimes\left(\widetilde{\mathbf{B}} G_{\mathbf{H}}(z)\right)\right]-\mathbb{E}\left[\left(G_{\mathbf{H}}(z) \widetilde{\mathbf{B}}\right) \otimes G_{\mathbf{H}}(z)\right]
$$

belongs to the kernel of the linear map $X \otimes Y \mapsto \tau(X) Y$. We shall prove that (64) belongs to the space:

$$
\begin{array}{r}
\left(\left(\begin{array}{ll}
\mathcal{M}_{N}(\mathbb{C}) & 0 \\
\mathcal{M}_{N}(\mathbb{C}) & 0
\end{array}\right) \otimes\left(\begin{array}{cc}
0 & 0 \\
\mathcal{M}_{N}(\mathbb{C}) & \mathcal{M}_{N}(\mathbb{C})
\end{array}\right)\right) \\
\oplus\left(\left(\begin{array}{ll}
0 & \mathcal{M}_{N}(\mathbb{C}) \\
0 & \mathcal{M}_{N}(\mathbb{C})
\end{array}\right) \otimes\left(\begin{array}{cc}
\mathcal{M}_{N}(\mathbb{C}) & \mathcal{M}_{N}(\mathbb{C}) \\
0 & 0
\end{array}\right)\right)
\end{array}
$$

which is of course enough. Let us define

$$
\Psi: \mathcal{M}_{2 N}(\mathbb{C}) \otimes \mathcal{M}_{2 N}(\mathbb{C}) \rightarrow \mathcal{L}\left(\mathcal{M}_{2 N}(\mathbb{C})\right)
$$


to be the linear map defined by $\Psi(X \otimes Y)(M)=X M Y$. It is easy to see that the space of (65) is precisely the space of elements of $T \in \mathcal{M}_{2 N}(\mathbb{C}) \otimes \mathcal{M}_{2 N}(\mathbb{C})$ such that

$$
\left(\begin{array}{cc}
\mathcal{M}_{N}(\mathbb{C}) & 0 \\
0 & \mathcal{M}_{N}(\mathbb{C})
\end{array}\right) \subset \operatorname{ker} \Psi(T) .
$$

Hence, it suffices to prove that for any $Z, Z^{\prime} \in \mathcal{M}_{N}(\mathbb{C})$,

$$
\mathbb{E}\left[G_{\mathbf{H}}(z)\left(\begin{array}{cc}
Z & 0 \\
0 & Z^{\prime}
\end{array}\right) \widetilde{\mathbf{B}} G_{\mathbf{H}}(z)-G_{\mathbf{H}}(z) \widetilde{\mathbf{B}}\left(\begin{array}{cc}
Z & 0 \\
0 & Z^{\prime}
\end{array}\right) G_{\mathbf{H}}(z)\right]=0 .
$$

By linearity, one can suppose that $Z, Z^{\prime}$ are Hermitian. Then one recognizes easily that the LHT of (66) is (up to a constant factor) the derivative, at $t=0$, of $\mathbb{E}\left[G_{\mathbf{H}_{t}}(z)\right]$, where

$$
\mathbf{H}_{t}:=\mathbf{A}+\left(\begin{array}{cc}
\mathrm{e}^{\mathrm{i} t Z} & 0 \\
0 & \mathrm{e}^{\mathrm{i} t Z^{\prime}}
\end{array}\right) \mathbf{W B} \mathbf{W}^{*}\left(\begin{array}{cc}
\mathrm{e}^{-\mathrm{i} t Z} & 0 \\
0 & \mathrm{e}^{-\mathrm{i} t Z^{\prime}}
\end{array}\right) .
$$

By invariance of the Haar measure, we have

$$
\left(\begin{array}{cc}
\mathrm{e}^{\mathrm{i} t Z} & 0 \\
0 & \mathrm{e}^{\mathrm{i} t Z^{\prime}}
\end{array}\right) \mathbf{W} \stackrel{\text { law }}{=} \mathbf{W},
$$

hence the above derivative is null. This proves the lemma.

Let

$$
S_{B}(z):=-\frac{\mathbb{E}\left[f_{B}(z)\right]}{\mathbb{E}\left[m_{\mathbf{H}}(z)\right]}
$$

for $f_{B}(z)$ and $m_{\mathbf{H}}(z)$ defined at (62) and (15). For $X, Y$ matrices, let $[X, Y]:=$ $X Y-Y X$ denote the commutant of $X$ and $Y$.

LEMMA 5.3. Let

$$
\begin{aligned}
\Delta_{A}(z)= & -\mathbb{E}\left[\tau\left(\stackrel{\circ}{G}_{\mathbf{H}}(z)\right) G_{\mathbf{H}}(z)\right] \\
& -\mathbb{E}\left[\left[\tau\left(\stackrel{\circ}{G}_{\mathbf{H}}(z)\right), G_{\mathbf{A}}(z)\right] \widetilde{\mathbf{B}} G_{\mathbf{H}}(z)\right]-\mathbb{E}\left[\tau\left(\stackrel{\circ}{G}_{\mathbf{H}}(z) \widetilde{\mathbf{B}}\right) G_{\mathbf{H}}(z)\right]
\end{aligned}
$$

and

$$
R_{A}(z):=\frac{G_{\mathbf{A}}\left(z+S_{B}(z)\right)(\mathbf{A}-z) \Delta_{A}(z)}{\mathbb{E}\left[m_{\mathbf{H}}(z)\right]} .
$$

Then we have

$$
\mathbb{E}\left[G_{\mathbf{H}}(z)\right]=G_{\mathbf{A}}\left(z+S_{\mathbf{B}}(z)\right)+R_{A}(z) .
$$


Proof. By Remark 5.1, $\mathbb{E}\left[\tau\left(G_{\mathbf{H}}(z)\right)\right]$ is a scalar $2 N \times 2 N$ matrix equal to $\mathbb{E}\left[m_{\mathbf{H}}(z)\right] I$. Thus, using successively (18), the resolvant identity and the previous lemma, we get

$$
\begin{aligned}
& \mathbb{E}\left[m_{\mathbf{H}}(z)\right] \mathbb{E}\left[G_{\mathbf{H}}(z)\right] \\
&=\mathbb{E}\left[\tau\left(G_{\mathbf{H}}(z)\right)\right] \mathbb{E}\left[G_{\mathbf{H}}(z)\right] \\
&=\mathbb{E}\left[\tau\left(G_{\mathbf{H}}(z)\right) G_{\mathbf{H}}(z)\right]-\underbrace{\mathbb{E}\left[\tau\left(\stackrel{\circ}{\mathbf{H}}_{\mathbf{H}}(z)\right) G_{\mathbf{H}}(z)\right]}_{:=\varepsilon_{1}} \\
&=\mathbb{E}\left[\tau\left(G_{\mathbf{H}}(z)\right)\left\{G_{\mathbf{A}}(z)-G_{\mathbf{A}}(z) \widetilde{\mathbf{B}} G_{\mathbf{H}}(z)\right\}\right]-\varepsilon_{1} \\
&=\mathbb{E}\left[m_{\mathbf{H}}(z)\right] G_{\mathbf{A}}(z)-\mathbb{E}\left[\tau\left(G_{\mathbf{H}}(z)\right) G_{\mathbf{A}}(z) \widetilde{\mathbf{B}} G_{\mathbf{H}}(z)\right]-\varepsilon_{1} \\
&= \mathbb{E}\left[m_{\mathbf{H}}(z)\right] G_{\mathbf{A}}(z)-\underbrace{\mathbb{E}\left[\left[\tau\left(G_{\mathbf{H}}(z)\right), G_{\mathbf{A}}(z)\right] \widetilde{\mathbf{B}} G_{\mathbf{H}}(z)\right]}_{:=\varepsilon_{2}} \\
&-\mathbb{E}\left[G_{\mathbf{A}}(z) \tau\left(G_{\mathbf{H}}(z)\right) \widetilde{\mathbf{B}} G_{\mathbf{H}}(z)\right]-\varepsilon_{1} \\
&= \mathbb{E}\left[m_{\mathbf{H}}(z)\right] G_{\mathbf{A}}(z)-G_{\mathbf{A}}(z) \mathbb{E}\left[\tau\left(G_{\mathbf{H}}(z) \widetilde{\mathbf{B}}\right) G_{\mathbf{H}}(z)\right]-\varepsilon_{1}-\varepsilon_{2} \\
&= \mathbb{E}\left[m_{\mathbf{H}}(z)\right] G_{\mathbf{A}}(z)-G_{\mathbf{A}}(z) \mathbb{E}\left[\tau\left(G_{\mathbf{H}}(z) \widetilde{\mathbf{B}}\right)\right] \mathbb{E}\left[G_{\mathbf{H}}(z)\right] \\
&-\underbrace{}_{:=\varepsilon_{\mathbf{A}}(z) \mathbb{E}\left[\tau\left(\stackrel{\circ}{H}_{\mathbf{H}}(z) \widetilde{\mathbf{B}}\right) G_{\mathbf{H}}(z)\right]-\varepsilon_{1}-\varepsilon_{2}} \\
&= \mathbb{E}\left[m_{\mathbf{H}}(z)\right] G_{\mathbf{A}}(z)-G_{\mathbf{A}}(z) \mathbb{E}\left[\tau\left(G_{\mathbf{H}}(z) \widetilde{\mathbf{B}}\right)\right] \mathbb{E}\left[G_{\mathbf{H}}(z)\right]-\varepsilon_{1}-\varepsilon_{2}-\varepsilon_{3} \\
&= \mathbb{E}\left[m_{\mathbf{H}}(z)\right] G_{\mathbf{A}}(z)-G_{\mathbf{A}}(z) \mathbb{E}\left[f_{B}(z)\right] \mathbb{E}\left[G_{\mathbf{H}}(z)\right]-\varepsilon_{1}-\varepsilon_{2}-\varepsilon_{3} .
\end{aligned}
$$

Dividing by the complex number $\mathbb{E}\left[m_{\mathbf{H}}(z)\right]$ and multiplying on the left by $\mathbf{A}-z$, one gets

$$
(\mathbf{A}-z) \mathbb{E}[G \mathbf{H}(z)]=I+S_{B}(z) \mathbb{E}\left[G_{\mathbf{H}}(z)\right]-\varepsilon_{1}^{\prime}-\varepsilon_{2}^{\prime}-\varepsilon_{3}^{\prime},
$$

for $\varepsilon_{i}^{\prime}:=\frac{(\mathbf{A}-z) \varepsilon_{i}}{\mathbb{E}\left[m_{\mathbf{H}}(z)\right]}$. This gives

$$
\left(\mathbf{A}-z-S_{B}(z)\right) \mathbb{E}\left[G_{\mathbf{H}}(z)\right]=I-\varepsilon_{1}^{\prime}-\varepsilon_{2}^{\prime}-\varepsilon_{3}^{\prime},
$$

that is,

$$
\mathbb{E}\left[G_{\mathbf{H}}(z)\right]=G_{\mathbf{A}}\left(z+S_{B}(z)\right)-\varepsilon_{1}^{\prime \prime}-\varepsilon_{2}^{\prime \prime}-\varepsilon_{3}^{\prime \prime},
$$

for

$$
\varepsilon_{i}^{\prime \prime}:=G_{\mathbf{A}}\left(z+S_{B}(z)\right) \varepsilon_{i}^{\prime}=\frac{G_{\mathbf{A}}\left(z+S_{B}(z)\right)(\mathbf{A}-z) \varepsilon_{i}}{\mathbb{E}\left[m_{\mathbf{H}}(z)\right]} .
$$

To conclude, it suffices to notice that

$$
R_{A}(z)=-\varepsilon_{1}^{\prime \prime}-\varepsilon_{2}^{\prime \prime}-\varepsilon_{3}^{\prime \prime},
$$

up to the fact that in the second term of $R_{A}(z)$, we have $\left[\tau\left(\stackrel{\circ}{G}_{\mathbf{H}}(z)\right), G_{\mathbf{A}}(z)\right]$ instead of $\left[\tau\left(G_{\mathbf{H}}(z)\right), G_{\mathbf{A}}(z)\right]$. But as $\mathbb{E}\left[\tau\left(G_{\mathbf{H}}(z)\right)\right]$ is a scalar matrix, both are equal. 
LEMMA 5.4. Let $\Psi_{A}(z):=\frac{1}{\mathbb{E} m_{\mathbf{H}}(z)}(\mathbf{A}-z) \mathbb{E}\left[\Delta_{A}(z)\right]$ and $Y_{A}(z):=(I+$ $\left.\Psi_{A}(z)\right)^{-1}-I$. Then

$$
S_{B}(z) I=-\left(\mathbb{E} G_{\mathbf{H}}(z)\right)^{-1}+\mathbf{A}-z+Y_{A}(z)\left(\mathbf{A}-z-S_{B}(z)\right),
$$

where I denotes the identity matrix.

ProOF. By the previous lemma,

$$
\begin{aligned}
\mathbb{E} G_{\mathbf{H}}(z) & =G_{\mathbf{A}}\left(z+S_{B}(z)\right)\left(I+\frac{1}{\mathbb{E} m_{\mathbf{H}}(z)}(\mathbf{A}-z) \mathbb{E} \Delta_{A}\right) \\
& =G_{\mathbf{A}}\left(z+S_{B}(z)\right)\left(I+\Psi_{A}(z)\right) \\
& =G_{\mathbf{A}}\left(z+S_{B}(z)\right)\left(I+Y_{A}(z)\right)^{-1}
\end{aligned}
$$

hence

$$
\left(\mathbb{E} G_{\mathbf{H}}(z)\right)^{-1}=\left(I+Y_{A}(z)\right)\left(\mathbf{A}-\left(z+S_{B}(z)\right)\right)
$$

which allows to conclude.

LEMMA 5.5. Let $\rho$ be a probability measure supported by $[-K, K]$ and $|z| \leq$ $K$. Then

$$
\operatorname{Im} m_{\rho}(z) \geq \frac{\eta}{5 K^{2}}
$$

PROOF. It suffices to note that for any $\lambda \in[-K, K], \operatorname{Im} \frac{1}{\lambda-z}=\frac{\eta}{(\lambda-E)^{2}+\eta^{2}} \geq$ $\frac{\eta}{(2 K)^{2}+K^{2}}$.

It follows from this lemma that there is $c$ depending only on $K$ such that

$$
\frac{1}{\left|\mathbb{E} m_{\mathbf{H}}(z)\right|} \leq \frac{c}{\eta}
$$

and

$$
\left|S_{B}(z)\right|=\left|\frac{\mathbb{E} f_{B}(z)}{\mathbb{E} m_{\mathbf{H}}(z)}\right| \leq \frac{c}{\eta^{2}} .
$$

LEMMA 5.6. For any $\delta>0$,

$$
\begin{array}{r}
\mathbb{P}\left(\left\|\tau\left(\stackrel{\circ}{G}_{\mathbf{H}}(z)\right) G_{\mathbf{H}}(z)\right\| \geq \delta\right) \leq 4 \exp \left(-c \frac{\delta^{2} \eta^{6}}{\|B\|^{2}} N^{2}\right), \\
\mathbb{P}\left(\left\|\left[\tau\left(\stackrel{\circ}{G}_{\mathbf{H}}(z)\right), G_{\mathbf{A}}(z)\right] \widetilde{\mathbf{B}} G_{\mathbf{H}}(z)\right\| \geq \delta\right) \leq 4 \exp \left(-c \frac{\delta^{2} \eta^{8}}{\|B\|^{4}} N^{2}\right)
\end{array}
$$

and

$$
\mathbb{P}\left(\left\|\tau\left(\stackrel{\circ}{G}_{\mathbf{H}}(z) \widetilde{\mathbf{B}}\right) G_{\mathbf{H}}(z)\right\| \geq \delta\right) \leq 4 \exp \left(-c \frac{\delta^{2} \eta^{6}}{\|B\|^{4}\left(1+\frac{\eta}{\|B\|}\right)^{2}} N^{2}\right)
$$


Proof. To prove it, as for any Hermitian matrix $M,\left\|G_{M}(z)\right\| \leq \eta^{-1}$, it suffices to prove that for any $\delta>0$,

$$
\mathbb{P}\left(\left\|\tau\left(\stackrel{\circ}{G}_{\mathbf{H}}(z)\right)\right\| \geq \delta\right) \leq 4 \exp \left(-c \frac{\delta^{2} \eta^{4}}{\|B\|^{2}} N^{2}\right)
$$

and

$$
\mathbb{P}\left(\left\|\tau\left(\stackrel{\circ}{G}_{\mathbf{H}}(z) \widetilde{\mathbf{B}}\right)\right\| \geq \delta\right) \leq 4 \exp \left(-c \frac{\delta^{2} \eta^{4}}{\|B\|^{4}\left(1+\frac{\eta}{\|B\|}\right)^{2}} N^{2}\right) .
$$

We shall apply the Lemma A.14 of the Appendix. Note first that a Haardistributed unitary matrix can be realized as the product of a Haar-distributed $\mathcal{S U}_{N}$ matrix by a uniform random phase, hence up to a randomization of $B$ by multiplication by an independent uniform phase, one can suppose that $U$ and $V$ are independent Haar-distributed $\mathcal{S U}_{N}$ matrices.

Let $P_{1}, P_{2}$ be the $2 N \times 2 N$ matrices defined by

$$
P_{1}:=\left(\begin{array}{cc}
I & 0 \\
0 & 0
\end{array}\right), \quad P_{2}:=\left(\begin{array}{cc}
0 & 0 \\
0 & I
\end{array}\right),
$$

so that for any $M \in \mathcal{M}_{2 N}(\mathbb{C})$,

$$
\tau(M)=\left(\begin{array}{cc}
N^{-1} \operatorname{Tr} P_{1} M P_{1} & 0 \\
0 & N^{-1} \operatorname{Tr} P_{2} M P_{2}
\end{array}\right) .
$$

Let $\varphi_{i}, \psi_{i}(i=1,2)$ be the functions defined on $\left(\mathcal{S U}_{N}\right)^{2}$ by

$$
\varphi_{i}(U, V):=N^{-1} \operatorname{Tr}\left(P_{i} G_{\mathbf{H}}(z) P_{i}\right), \quad \psi_{i}(U, V):=N^{-1} \operatorname{Tr}\left(P_{i} G_{\mathbf{H}}(z) \widetilde{\mathbf{B}} P_{i}\right)
$$

with the notation of (22), (23). We need to prove that under the sole hypothesis that $\|A\|,\|B\|,|z| \leq K$, the numbers

$$
\frac{N L_{\varphi_{i}}^{2} \eta^{4}}{\|B\|^{2}} ; \quad \frac{N L_{\psi_{i}}^{2} \eta^{4}}{\|B\|^{4}\left(1+\frac{\eta}{\|B\|}\right)^{2}} ; \quad(i=1,2)
$$

are bounded uniformly in $N$.

For $X, Y$ skew-Hermitian matrices with null traces and $U, V \in \mathcal{S} \mathcal{U}_{N}$,

$$
\partial_{t, t=0} \varphi_{i}\left(\mathrm{e}^{t X} U, \mathrm{e}^{t Y} V\right)=-N^{-1} \operatorname{Tr}\left(P_{i} G_{\mathbf{H}}(z)(\mathbf{Z} \widetilde{\mathbf{B}}-\widetilde{\mathbf{B Z}}) G_{\mathbf{H}}(z) P_{i}\right),
$$

with $\mathbf{Z}:=\left(\begin{array}{cc}X & 0 \\ 0 & Y\end{array}\right)$, so that

$$
\nabla \varphi_{i}(U, V)=-\frac{1}{N} \mathbf{W}^{*} \mathcal{P}\left(\left[\widetilde{\mathbf{B}}, G_{\mathbf{H}}(z) P_{i} G_{\mathbf{H}}(z)\right]\right),
$$

where $\mathcal{P}$ is the orthogonal projection from $\mathcal{M}_{2 N}(\mathbb{C})$ onto the tangent space at $I$, of $\left(\mathcal{S U}_{N}\right)^{2}$. As this projection does not enlarge the norm, the usual noncommutative Hôlder inequalities (see Appendix A.3 of [1]) allow to claim that

$$
\frac{N L_{\varphi_{i}}^{2} \eta^{4}}{\|B\|^{2}}
$$


is bounded.

In the same way,

$$
\begin{aligned}
\partial_{t, t=0} \psi_{i}\left(\mathrm{e}^{t X} U, \mathrm{e}^{t Y} V\right) \\
\quad=N^{-1} \operatorname{Tr}\left\{P_{i}\left(-G_{\mathbf{H}}(z)(\mathbf{Z} \widetilde{\mathbf{B}}-\widetilde{\mathbf{B} Z}) G_{\mathbf{H}}(z) \widetilde{\mathbf{B}}+G_{\mathbf{H}}(z)(\mathbf{Z} \widetilde{\mathbf{B}}-\widetilde{\mathbf{B} Z})\right) P_{i}\right\},
\end{aligned}
$$

hence

$$
\nabla \varphi_{i}(U, V)=\frac{1}{N} W^{*} \mathcal{P}\left(\left[\widetilde{\mathbf{B}},-G_{\mathbf{H}}(z) \widetilde{\mathbf{B}} P_{i} G_{\mathbf{H}}(z)+P_{i} G_{\mathbf{H}}(z)\right]\right),
$$

and one concludes as above.

LEMMA 5.7. We have

$$
\begin{aligned}
\mathbb{E}\left\|\tau\left(\stackrel{\circ}{G}_{\mathbf{H}}(z)\right) G_{\mathbf{H}}(z)\right\| & \leq c \frac{\|B\|}{N \eta^{3}}, \\
\mathbb{E}\left\|\left[\tau\left(\stackrel{\circ}{G}_{\mathbf{H}}(z)\right), G_{\mathbf{A}}(z)\right] \widetilde{\mathbf{B}} G_{\mathbf{H}}(z)\right\| & \leq c \frac{\|B\|^{2}}{N \eta^{4}}
\end{aligned}
$$

and

$$
\mathbb{E}\left\|\tau\left(\stackrel{\circ}{G}_{\mathbf{H}}(z) \widetilde{\mathbf{B}}\right) G_{\mathbf{H}}(z)\right\| \leq c \frac{\|B\|^{2}\left(1+\frac{\eta}{\|B\|}\right)}{N \eta^{3}} .
$$

Hence, if $|z|,\|B\| \leq K$, then

$$
\left\|\Delta_{A}(z)\right\| \leq c \frac{1}{N \eta^{4}} ; \quad\left\|\Psi_{A}(z)\right\| \leq c \frac{1}{N \eta^{5}}
$$

and

$$
N \eta^{5} \geq 2 c \quad \Longrightarrow \quad\left\|Y_{A}(z)\right\| \leq \frac{2 c}{N \eta^{5}} .
$$

PROOF. The three first inequalities follow from the previous lemma and standard queues-moments relations. The upper bound on $\left\|\Delta_{A}(z)\right\|$ follows from the very definition of $\Delta_{A}(z)$ at Lemma 5.3. The upper bound on $\left\|\Psi_{A}(z)\right\|$ follows from its definition

$$
\Psi_{A}(z)=\frac{1}{\mathbb{E} m_{\mathbf{H}}(z)}(\mathbf{A}-z) \mathbb{E}\left[\Delta_{A}(z)\right]
$$

and from (70). At last, (73) follows from the definition $Y_{A}(z)=\left(I+\Psi_{A}(z)\right)^{-1}-I$ and from the well-known inequality:

$$
\|X\| \leq 1 / 2 \quad \Longrightarrow \quad\left\|(I-X)^{-1}-I\right\| \leq 2\|X\| .
$$

Adapting the proof of Lemma 4.7 of [9], we get the following lemma. 
LEMMA 5.8. Let $\mathcal{U} \subset \mathcal{M}_{N}(\mathbb{C})$ be a compact Lie group and let $\mathcal{L}$ be the complex linear subspace of $\mathcal{M}_{N}(\mathbb{C})$ spanned by its Lie algebra. Let us fix $M \in \mathcal{M}_{N}(\mathbb{C})$ and, for $b \in \mathcal{M}_{N}(\mathbb{C})$ such that $b-u M u^{-1}$ is invertible for any $u \in \mathcal{U}$, define the random matrix $R(b):=\left(b-U M U^{-1}\right)^{-1}$, where $U$ is Haar-distributed in $\mathcal{U}$. Then:

(i) for any $Y \in \mathcal{L}$, we have $\mathbb{E}[R(b)] Y-Y \mathbb{E}[R(b)]=\mathbb{E}[R(b)(Y b-b Y) R(b)]$,

(ii) the matrix $\mathbb{E}[R(b)]$ commutes with any matrix in $\mathcal{L}$ commuting with $b$.

Proof. (ii) is a direct consequence of (i). Besides, by linearity, it suffices to prove (i) for $Y$ in the Lie algebra of $\mathcal{U}$. For such a matrix $Y$, differentiating at 0 the constant function $f(t):=\mathbb{E}\left[\left(b-\mathrm{e}^{t Y} U M U^{-1} \mathrm{e}^{-t Y}\right)^{-1}\right]$, we get

$$
\mathbb{E}\left[R(b)\left(U M U^{-1} Y-Y U M U^{-1}\right) R(b)\right]=0 .
$$

Then, using $R(b) U M U^{-1}=-I+R(b) b$ and $U B U^{-1} R(b)=-I+b R(b)$, we get (i) directly.

\section{LEMMA 5.9. The matrix $\mathbb{E} G_{\mathbf{H}}(z)$ commutes with A.}

PROOF. Let us apply the previous lemma for $\mathcal{U}$ the group of matrices $\left(\begin{array}{cc}U & 0 \\ 0 & V\end{array}\right)$, for $U, V$ unitary matrices (so that $\mathcal{L}$ is the space of matrices $\left(\begin{array}{cc}X & 0 \\ 0 & X^{\prime}\end{array}\right)$, for $X, X^{\prime} \in$ $\left.\mathcal{M}_{N}(\mathbb{C})\right), M=\mathbf{B}$ and $b=z-\mathbf{A}$. It states that $\mathbb{E} G_{\mathbf{H}}(z)$ commutes with any matrix of $\mathcal{L}$ commuting with $\mathbf{A}$, for example with any matrix of the type $\left(\begin{array}{cc}D & 0 \\ 0 & D\end{array}\right)$, with $D$ diagonal. We deduce that

$$
\mathbb{E} G_{\mathbf{H}}(z)=\left(\begin{array}{cc}
J & K \\
L & M
\end{array}\right)
$$

with $J, K, L, M$ some $N \times N$ diagonal matrices. But by Remark 5.1, the matrix $\mathbb{E} G_{\mathbf{H}}(z)$ is invariant under conjugation by the matrix $P$ introduced at (60). By (61), it implies that $J=M$ and $K=L$. It suffices to conclude.

5.2. Proof of Theorem 1.5 (Matrix subordination). Note first that the statement is symmetric in $A$ and $B$, so we shall prove it for $S_{B}$ and $R_{A}$ only.

By Lemmas 5.3 and 5.4, we have

$$
\mathbb{E}\left[G_{\mathbf{H}}(z)\right]=G_{\mathbf{A}}\left(z+S_{\mathbf{B}}(z)\right)+R_{A}(z)
$$

with

$$
R_{A}(z):=\frac{G_{\mathbf{A}}\left(z+S_{B}(z)\right)(\mathbf{A}-z) \Delta_{A}(z)}{\mathbb{E}\left[m_{\mathbf{H}}(z)\right]}
$$

and

$$
S_{B}(z) I=-\left(\mathbb{E} G_{\mathbf{H}}(z)\right)^{-1}+\mathbf{A}-z+Y_{A}(z)\left(\mathbf{A}-z-S_{B}(z)\right)
$$


By Proposition 2 of [27], we know that

$$
-\left(\mathbb{E} G_{\mathbf{H}}(z)\right)^{-1}
$$

has all its eigenvalues with imaginary part $\geq \eta$. But by Lemma 5.9, $\mathbb{E} G_{\mathbf{H}}(z)$ commutes with $\mathbf{A}$, hence the eigenvalues of $-\left(\mathbb{E} G_{\mathbf{H}}(z)\right)^{-1}+\mathbf{A}-z$ have nonnegative imaginary parts. Thus, by standard perturbation analysis (see, e.g., [18], Chapter 4),

$$
\operatorname{Im} S_{B}(z) \geq\left\|Y_{A}(z)\left(\mathbf{A}-z-S_{B}(z)\right)\right\| .
$$

Then (71) and (73) give directly the lower-bound (26) on the imaginary part of $S_{B}(z)$.

The upper bound (27) on $\left\|R_{A}(z)\right\|$ follows directly from the expression (74), the upper bound (72) on $\left\|\Delta_{A}(z)\right\|$, and the fact that for $N$ large enough, $\operatorname{Im}(z+$ $\left.S_{B}(z)\right) \geq \frac{\eta}{2}$.

\section{APPENDIX}

A.1. Free convolution and subordination. Let us first recall one of the ways to define the free convolution $[5,8,14]$.

THEOREM A.10 (Definition of the free convolution via subordination). Let $\mu, v$ be probability measures on the real line with compact supports. Then the system

$$
\begin{aligned}
m(z) & =m_{\mu}\left(z+S_{v}(z)\right), \\
m(z) & =m_{v}\left(z+S_{\mu}(z)\right), \\
-\left(z+\frac{1}{m(z)}\right) & =S_{\mu}(z)+S_{v}(z)
\end{aligned}
$$

has a unique solution $\left(m(\cdot), S_{\mu}(\cdot), S_{\nu}(\cdot)\right)$ in the class of triplets of analytic functions on $\mathbb{C}^{+}$satisfying, as $|z| \rightarrow+\infty$,

$$
\begin{aligned}
m(z) & =-z^{-1}+O\left(z^{-2}\right), \\
\left|S_{\mu}(z)\right|+\left|S_{\nu}(z)\right| & =O(1) .
\end{aligned}
$$

The function $m(z)$ is then the Stieltjes' transform of a unique probability measure, which is $\mu \boxplus v$. Moreover, $S_{\mu}$ and $S_{v}$ take values in $\overline{\mathbb{C}^{+}}$.

REMARK A.11. Note that this result, in addition to define the free convolution, is a first regularity result for this convolution. Indeed, for any $\eta>0$ and any probability measure $\rho$ on the real line, the function $z \mapsto m_{\rho}(z+\mathrm{i} \eta)$ is the Stieltjes' transform of an analytic regularization of $\rho$ (namely its classical convolution with 
the Cauchy law $\left.\frac{1}{\pi} \frac{\eta \mathrm{d} x}{x^{2}+\eta^{2}}\right)$. Hence, for $\mu, \rho$ some probability measures on the real line, the equation

$$
m_{\rho}(z)=m_{\mu}\left(z^{\prime}\right) \quad \text { with } \operatorname{Im} z^{\prime}>\operatorname{Im} z
$$

implies roughly that $\rho$ is more regular than $\mu$.

The following lemmas will be used in this text.

LEMMA A.12. Let $X, Y$ be free self-adjoint elements of a tracial $W^{*}$ probability space $(\mathcal{A}, \tau)$ with respective distributions $\mu, v$. Then for any $z \in \mathbb{C}^{+}$, we have

$$
S_{\mu}(z)=-\frac{\tau\left(X(X+Y-z)^{-1}\right)}{\tau\left((X+Y-z)^{-1}\right)} ; \quad S_{\nu}(z)=-\frac{\tau\left(Y(X+Y-z)^{-1}\right)}{\tau\left((X+Y-z)^{-1}\right)} .
$$

PROOF. Let us focus for example on $S_{\mu}$. It is equivalent to prove that

$$
z+S_{\mu}(z)=z-\frac{\tau\left(X(X+Y-z)^{-1}\right)}{\tau\left((X+Y-z)^{-1}\right)}=\frac{\tau\left((z-X)(X+Y-z)^{-1}\right)}{\tau\left((X+Y-z)^{-1}\right)}
$$

that is,

$$
\left(z+S_{\mu}(z)\right) \tau\left((X+Y-z)^{-1}\right)=\tau\left((z-X)(X+Y-z)^{-1}\right) .
$$

Let $\tau_{Y}$ denote the conditional (noncommutative) expectation given the $W^{*}$-algebra generated by $Y$. We know, by Theorem 3.1 of [14], that

$$
\tau_{Y}\left(\frac{1}{z-X-Y}\right)=\frac{1}{z+S_{\mu}(z)-Y},
$$

so that

$$
z+S_{\mu}(z)=Y+\left(\tau_{Y}\left(\frac{1}{z-X-Y}\right)\right)^{-1}
$$

(the miracle of [14] being precisely that despite the $\tau_{Y}$ in the RHT, $z+S_{\mu}(z)$ is a scalar) and

$$
\left(z+S_{\mu}(z)\right) \tau_{Y}\left(\frac{1}{z-X-Y}\right)=Y \tau_{Y}\left(\frac{1}{z-X-Y}\right)+1 .
$$

Let us now apply $\tau$. As $z+S_{\mu}(z) \in \mathbb{C}$, we get

$$
\left(z+S_{\mu}(z)\right) \tau\left((z-X-Y)^{-1}\right)=\tau\left(Y(z-X-Y)^{-1}\right)+1,
$$

which is exactly (76). 
LEMMA A.13. Let $K>0$ be fixed. Then there are $M_{i}=M_{i}(K)>0(i=1,2)$ such that for any pair $\mu, v$ of probability measures with supports contained in $[-K, K]$, for any $|z|>M_{1}$, the numbers $S_{\mu^{s}}(z), S_{\nu^{s}}(z)$ (defined at Theorem A.10), $\kappa_{\mu^{s}, v^{s}}(z), \alpha_{\mu^{s}, v^{s}}(z)$ and $\beta_{\mu^{s}, v^{s}}(z)$ [defined at (31), (32) and (33)] satisfy

$$
\begin{aligned}
\kappa_{\mu^{s}, v^{s}}(z) & \geq \frac{1}{M_{2}} ; \quad \frac{1}{\alpha_{\mu^{s}, \nu^{s}}(z) \beta_{\mu^{s}, \nu^{s}}(z)} \leq M_{2} ; \\
\frac{\alpha_{\mu^{s}, \nu^{s}}(z)}{\left|z+S_{\mu^{s}}(z)+S_{\nu^{s}}(z)\right|^{2}} & \leq M_{2} .
\end{aligned}
$$

PROOF. First, by the previous lemma, we know that the estimate (76) is uniform in all pairs $\mu, v$ of probability measures with supports contained in $[-K, K]$. Besides, it is obvious, from the series expansion, that the estimates $-z^{k} m_{\mu}^{(k)}(z) \longrightarrow 1, k=0,1,2$, as $|z| \longrightarrow \infty$, are uniform in probability measures $\mu$ with support contained in $[-K, K]$. Then, going back to the formulas defining the functions of interest here, we get the desired estimates.

A.2. Concentration of measure for the Haar measure. By the lemma of Gromov and Milman (see, e.g., [1], page 299) and Proposition 1.11 of [29], we have the following.

LEMMA A.14. Let $f$ be a smooth real-valued function on $\left(\mathcal{S U}_{N}\right)^{2}$ and let

$$
L_{f}:=\max _{(U, V) \in\left(\mathcal{S} \mathcal{U}_{N}\right)^{2}} \sqrt{\operatorname{Tr}\left(\nabla f(U, V) \nabla f(U, V)^{*}\right)} .
$$

Then for $U, V$ independent Haar-distributed $\mathcal{S U}_{N}$ matrices, for any $\delta \geq 0$,

$$
\mathbb{P}(|f(U, V)-\mathbb{E}[f(U, V)]| \geq \delta) \leq 2 \exp \left(-\frac{N \delta^{2}}{4 L_{f}^{2}}\right)
$$

A.3. Kantorovich's theorem on Newton's method. Let us give the simplified version of Kantorovich's theorem that we need (particular case of [21], Theorem 1). We let $\|\cdot\|$ denote the canonical Euclidian norm on $\mathbb{R}^{d}$ or the associated operator norm on $\mathcal{L}\left(\mathbb{R}^{d}\right)$.

THEOREM A.15. Let $O$ be an open subset of $\mathbb{R}^{d}$ and $F: O \rightarrow \mathbb{R}^{d}$ be a $\mathcal{C}^{1}$ function. Let $x_{0} \in O$ such that $F^{\prime}\left(x_{0}\right)$ is invertible and $y_{0} \in \mathbb{R}^{d}$. Suppose that for

$$
L:=\sup _{x \neq y \in O} \frac{\left\|F^{\prime}\left(x_{0}\right)^{-1}\left(F^{\prime}(x)-F^{\prime}(y)\right)\right\|}{\|x-y\|} ; \quad b:=\left\|F^{\prime}\left(x_{0}\right)^{-1}\left(F\left(x_{0}\right)-y_{0}\right)\right\|
$$

we have $2 b L<1$. Define

$$
r_{*}:=\frac{2 b}{1+\sqrt{1-2 b L}} \leq 2 b ; \quad r_{* *}:=\frac{1+\sqrt{1-2 b L}}{L}
$$


and choose $\rho \in\left[r_{*}, r_{* *}\right)$ such that $\bar{B}\left(x_{0}, \rho\right) \subset O$. Then the equation $F(x)=y_{0}$ has a unique solution $x_{*}$ in $B\left(x_{0}, \rho\right)$ and this solution satisfies

$$
\left\|x_{*}-x_{0}\right\| \leq r_{*}
$$

\section{A.4. Local laws and Stieltjes' transforms.}

\section{A.4.1. Density and upper bound on the Stieltjes' transform.}

LEMMA A.16. For $\mu$ probability measure on the real line and $M>0$, we have equivalence between:

(i) $\mu$ admits a density $\rho$ with respect to the Lebesgue measure such that $\|\rho\|_{\infty} \leq M$

(ii) $\operatorname{Im} m_{\mu}$ is uniformly bounded, on $\mathbb{C}^{+}$, by $\pi M$.

Moreover, in this case, for any probability measure $v$ on $\mathbb{R}, \mu \boxplus v$ also admits a density with respect to the Lebesgue measure which is bounded by $M$.

Proof. For $z=E+\mathrm{i} \eta(E \in \mathbb{R}, \eta>0)$, we have

$$
\frac{1}{\pi} \operatorname{Im} m_{\mu}(z)=\int_{\lambda \in \mathbb{R}} \frac{\eta}{(E-\lambda)^{2}+\eta^{2}} \mathrm{~d} \mu(\lambda) .
$$

Hence if (i) holds, then

$$
\operatorname{Im} m_{\mu}(z)=\int_{\lambda \in \mathbb{R}} \frac{\eta}{(E-\lambda)^{2}+\eta^{2}} \rho(\lambda) \mathrm{d} \lambda \leq M \int_{\lambda \in \mathbb{R}} \frac{\eta}{(E-\lambda)^{2}+\eta^{2}} \mathrm{~d} \lambda \leq M \pi .
$$

Reciprocally, let us suppose that $\operatorname{Im} m_{\mu}$ is uniformly bounded, on $\mathbb{C}^{+}$, by $\pi M$. The law

$$
\frac{1}{\pi} \operatorname{Im} m_{\mu}(\lambda+\mathrm{i} \eta) \mathrm{d} \lambda=\mu * \frac{\eta \mathrm{d} x}{\pi\left(x^{2}+\eta^{2}\right)}
$$

converges weakly to $\mu$ as $\eta \downarrow 0$, hence for any $x<y$,

$$
\mu([x, y])=\lim _{\eta \rightarrow 0} \frac{1}{\pi} \int_{x}^{y} \operatorname{Im} m_{\mu}(\lambda+\mathrm{i} \eta) \mathrm{d} \lambda
$$

(indeed, by [5], Lemma 2.17, (2), $\mu$ has no atom), so for any $x<y, \mu([x, y]) \leq$ $M(y-x)$. This implies that the cumulative distribution function of $\mu$ is $M$-Lipschitz, hence is almost everywhere differentiable, with derivative $\leq M$ and is the integral of its derivative, which is exactly (i).

The last statement follows from the subordination for $\boxplus$ : by Theorem A.10, there is a function $S: \mathbb{C}^{+} \rightarrow \overline{\mathbb{C}^{+}}$such that on $\mathbb{C}^{+}, m_{\mu \boxplus v}(z)=m_{\mu}(z+S(z))$, which allows directly to conclude by what precedes. 


\section{A.4.2. Erdôs, Schlein and Yau's method.}

THEOREM A.17 (Erdős, Schlein, Yau). Let $v$ be a signed measure on $\mathbb{R}, E \in$ $\mathbb{R}$ and $\eta, M>0$. Then $\frac{|v([E \pm M \eta])|}{2 M \eta}$ is upper-bounded by

$$
\begin{aligned}
C\left(\left\|m_{\nu}(\cdot+\mathrm{i} \eta)\right\|_{\infty,[E \pm M \eta]}+\frac{|\nu|([E \pm 2 M \eta])}{M^{3 / 2} \eta}\right. & \\
& \left.+\frac{|v|([E-2 M \eta \pm \sqrt{M} \eta] \cup[E+2 M \eta \pm \sqrt{M} \eta])}{M \eta}+\frac{\operatorname{Im} m_{|\nu|}(E+\mathrm{i} M \eta)}{M}\right),
\end{aligned}
$$

for a certain universal constant $C$.

PROOF. Let us briefly present the ideas of the proof of Corollary 4.2 in [19]. It suffices to notice that for $R(\lambda):=\frac{1}{\pi} \int_{[E \pm M \eta]} \frac{\eta}{(x-\lambda)^{2}+\eta^{2}} \mathrm{~d} x$, there are some functions $T_{1}, T_{2}, T_{3}$ such that $R(\lambda)=\mathbb{1}_{|\lambda-E| \leq \eta M}+T_{1}(\lambda)+T_{2}(\lambda)+T_{3}(\lambda)$, with:

- $\left\|T_{1}\right\|_{\infty} \leq \frac{c}{\sqrt{M}}$ and $\operatorname{supp}\left(T_{1}\right) \subset[E \pm 2 M \eta]$,

- $\left\|T_{2}\right\|_{\infty} \leq 1$ and $\operatorname{supp}\left(T_{2}\right) \subset[E-2 M \eta \pm \sqrt{M} \eta] \cup[E+2 M \eta \pm \sqrt{M} \eta]$,

- $\left|T_{3}(\lambda)\right| \leq \frac{C M \eta^{2}}{(\lambda-E)^{2}+M^{2} \eta^{2}}$.

Hence, as $\int_{\lambda \in \mathbb{R}} R(\lambda) \mathrm{d} v(\lambda)=\frac{1}{\pi} \int_{[E \pm M \eta]} \operatorname{Im} m_{v}(x+\mathrm{i} \eta) \mathrm{d} x$, we have

$$
\begin{aligned}
v([E \pm M \eta])= & \frac{1}{\pi} \int_{[E \pm M \eta]} \operatorname{Im} m_{v}(x+i \eta) \mathrm{d} x \\
& -\int_{\lambda \in \mathbb{R}}\left(T_{1}(\lambda)+T_{2}(\lambda)+T_{3}(\lambda)\right) \mathrm{d} v(\lambda),
\end{aligned}
$$

which proves the theorem.

A.4.3. Helffer-Sjöstrand functional calculus. The use of this method in random matrix theory is quite recent (see [1], Proof of Lemma 5.5.5, or [20], Proof of Lemma B.1). As we shall use it in a noncommon scale (see Corollary A.19), we state it precisely here.

THEOREM A.18. Let $v$ a signed measure on $\mathbb{R}, p \geq 1$ and $\phi$ a $\mathcal{C}^{p+1}$ compactly supported function on the real line. Suppose that there are $\eta_{\min }, \delta>0$, $\alpha \in[0, p+1)$ such that

$$
\left(E \in \operatorname{supp}(\phi) \text { and } \eta>\eta_{\text {min }}\right) \quad \Longrightarrow \quad\left|m_{v}(E+\mathrm{i} \eta)\right| \leq \delta \eta^{-\alpha} .
$$

Then

$$
\left|\int \phi(x) v(\mathrm{~d} x)\right| \leq \frac{L\left\|\phi^{(p+1)}\right\|_{\infty}}{p ! \pi}|v|(\mathbb{R})\left(\frac{\eta_{\min }^{p}}{p}+\frac{\delta}{p-\alpha+1}\right),
$$

for $L$ the Lebesgue measure of the support of $\phi$. 
PROOF. - The function

$$
f_{v}:(E, \eta) \in \mathbb{R} \times[0,+\infty) \longmapsto \begin{cases}\eta m_{v}(E+\mathrm{i} \eta) & \text { if } \eta>0, \\ \mathrm{i} v(\{E\}) & \text { if } \eta=0,\end{cases}
$$

satisfies $\left\|f_{v}\right\|_{\infty} \leq|v|(\mathbb{R})$. Indeed, for $\eta>0$, we have

$$
\left|f_{v}(E, \eta)\right|=\left|\int \frac{\mathrm{d} v(x)}{x-\frac{E}{\eta}+\mathrm{i} \eta}\right| \leq \int \mathrm{d}|v|(x) .
$$

- Choose $\varphi: \mathbb{R} \rightarrow[0,1]$ a smooth function with value 1 in a neighborhood of 0 and supported by $[-1,1]$. Then set $\Psi(x+\mathrm{i} y):=\sum_{\ell=0}^{p} \frac{\mathrm{i}_{\ell !}^{\ell}}{\ell(\ell)}(x) \varphi(y) y^{\ell}$. Note that for $\bar{\partial}:=\partial_{x}+\mathrm{i} \partial_{y}$, the function

$$
(E, \eta) \in \mathbb{R} \times[0,+\infty) \longmapsto \begin{cases}\frac{\bar{\partial} \Psi(E+\mathrm{i} \eta)}{\eta^{p}} & \text { if } \eta>0, \\ \frac{\mathrm{i}^{p}}{p !} \phi^{(p+1)}(E) \varphi(0) & \text { if } \eta=0,\end{cases}
$$

is continuous and bounded. Indeed, we have

$$
\bar{\partial} \Psi(E+\mathrm{i} \eta)=\frac{\mathrm{i}^{p}}{p !} \phi^{(p+1)}(E) \varphi(\eta) \eta^{p}
$$

so that the continuity is obvious and

$$
\sup _{E \in \mathbb{R}, \eta>0}\left|\frac{\bar{\partial} \Psi(E+\mathrm{i} \eta)}{\eta^{p}}\right| \leq \frac{1}{p !}\left\|\phi^{(p+1)}\right\|_{\infty} .
$$

- Using this remark in the particular case where $p=1$, we get

$$
\begin{aligned}
& \int_{t \in \mathbb{R}} \Psi(t) \mathrm{d} v(t) \\
&=\pi^{-1} \operatorname{Re}\left(\iint_{(E, \eta) \in \mathbb{R} \times[0,+\infty)} \frac{\bar{\partial} \Psi(E+\mathrm{i} \eta)}{\eta} \eta m_{v}(E+\mathrm{i} \eta) \mathrm{d} E \mathrm{~d} \eta\right) .
\end{aligned}
$$

Indeed, (82) is continuous in $v$ (for the topology defined by bounded continuous functions) and linear in $v$, so it suffices to prove it for $v=\delta_{\lambda}$, with $\lambda \in \mathbb{R}$. Then it is the content of [12], Proposition C.1.

- As a consequence, as $\phi$ and $\Psi$ coincide on $\mathbb{R}$, using (82), (77) and (81), we get

$$
\begin{aligned}
\left|\int \phi(x) \nu(\mathrm{d} x)\right| & \leq \pi^{-1}\left|\iint_{(E, \eta) \in \operatorname{supp}(\phi) \times(0,1]} \frac{\bar{\partial} \Psi(E+\mathrm{i} \eta)}{\eta^{p}} \eta^{p} m_{\nu}(E+\mathrm{i} \eta) \mathrm{d} E \mathrm{~d} \eta\right| \\
& \leq(p ! \pi)^{-1} L\left\|\phi^{(p+1)}\right\|_{\infty}|v|(\mathbb{R})\left(\int_{0}^{\eta_{\min }} \eta^{p-1} \mathrm{~d} \eta+\delta \int_{0}^{1} \eta^{p-\alpha} \mathrm{d} \eta\right) \\
& \leq(p ! \pi)^{-1} L\left\|\phi^{(p+1)}\right\|_{\infty}|v|(\mathbb{R})\left(\frac{\eta_{\min }^{p}}{p}+\frac{\delta}{p-\alpha+1}\right) .
\end{aligned}
$$


COROllary A.19. Let $p \geq 1$, let $v_{N}$ be a sequence of signed measures. Suppose that for some constants $C, D, c>0$, we have

$$
\sup \left\{\left|m_{v_{N}}(z)\right| ;|\operatorname{Re}(z)| \leq K, \frac{C}{\sqrt{\log N}} \leq \operatorname{Im} z \leq D\right\} \leq C \mathrm{e}^{-c \sqrt{\log N}} .
$$

Let $\phi_{N}$ be a sequence of smooth compactly supported functions. Then there is $C^{\prime}=C^{\prime}(c, C)$ such that

$$
\left|v_{N}\left(\phi_{N}\right)\right| \leq \frac{C^{\prime}\left|v_{N}\right|(\mathbb{R}) L_{N}\left\|\phi_{N}^{(p+1)}\right\|_{\infty}}{(p+1) !(\log N)^{p / 2}}
$$

where $L_{N}$ is the Lebesgue measure of the support of $\phi_{N}$.

PROOF. We apply Theorem A.18 with $\eta_{\min }=\frac{C}{\sqrt{\log N}}, \delta=C \mathrm{e}^{-c \sqrt{\log N}}, \alpha=0$.

A.4.4. An application of Hadamard's three circles theorem. The following use of Hadamard's three circles theorem is due to Kargin, in [26]. All ideas of the proof of Theorem A.20 can be found in [26], but as it is not stated clearly, we give a short proof.

THEOREM A.20. Let $a>0$. There is $\delta_{0}=\delta_{0}(a)>0$ such that for all $\delta \in$ $\left(0, \delta_{0}\right)$, for all signed measure $v$,

$$
\begin{gathered}
\sup \left\{\left|m_{v}(z)\right| ; z=\mathrm{i} a \frac{\mathrm{e}+\mathrm{e}^{\mathrm{i} \theta}}{\mathrm{e}-\mathrm{e}^{\mathrm{i} \theta}}, \theta \in[0,2 \pi]\right\} \leq \delta \\
\Longrightarrow \sup _{z \in H_{a, r(\delta)}}\left|m_{v}(z)\right| \leq \mathrm{e}^{-\sqrt{-c \log \delta}},
\end{gathered}
$$

where $c:=2|\nu|(\mathbb{R}) / a, r(\delta):=\mathrm{e}^{-4 \sqrt{-c / \log \delta}}$ and for $a, r>0, H_{a, r}$ denotes the disc with diameter $\left[\mathrm{i} a \frac{1-r}{1+r}, \mathrm{i} a \frac{1+r}{1-r}\right] \subset \mathbb{C}^{+}$, that is, the disc with center $\mathrm{i} a \frac{1+r^{2}}{1-r^{2}}$ and radius $a \frac{2 r}{1-r^{2}}$.

PROOF. The starting point of the proof is the so-called Hadamard three circles theorem [25], stating that for $f: \mathbb{D}:=\{\xi \in \mathbb{C} ;|\xi|<1\} \rightarrow \mathbb{C}$ analytic, the function $M(r):=\sup _{|\xi|=r}|f(\xi)|$ is nondecreasing and the function $\widetilde{M}(s):=\log \left(M\left(\mathrm{e}^{s}\right)\right)$ is convex on $(-\infty, 0)$. If we suppose moreover that $f$ is such that for a certain constant $c$, for all $r<1$,

$$
(1-r) M(r) \leq c
$$

then there is $\delta_{0}=\delta_{0}(c)>0$ such that for all $\delta \in\left(0, \delta_{0}\right)$, we have

$$
M\left(\mathrm{e}^{-1}\right) \leq \delta \quad \Longrightarrow \quad M\left(r_{c}(\delta)\right) \leq \varepsilon_{c}(\delta),
$$


with $r_{c}(\delta):=\mathrm{e}^{-4 \sqrt{-c / \log \delta}}$ and $\varepsilon_{c}(\delta):=\mathrm{e}^{-\sqrt{-c \log \delta}}$. Indeed, it is equivalent to prove that there is $m_{0}=m_{0}(c)<0$ such that for all $m<m_{0}$, we have

$$
\widetilde{M}(-1) \leq m \quad \Longrightarrow \quad \widetilde{M}(-4 \sqrt{c /|m|}) \leq-\sqrt{c|m|},
$$

which follows from the convexity of $\widetilde{M}$ (applied at $-1<-4 \sqrt{c /|m|}<-\sqrt{c /|m|}$ ).

Then one concludes by noticing that for $a, v$ as in the statement of the theorem and $f(\xi):=m_{\nu}\left(\mathrm{i} a \frac{1+\xi}{1-\xi}\right),(83)$ is satisfied for $c:=2|\nu|(\mathbb{R}) / a$.

COROLLARY A.21. Let $\mu_{N}, \mu$ be probability measures such that for a certain $a>0$ and a certain $C>0$, we have

$$
\sup \left\{\left|m_{\mu_{N}-\mu}(z)\right| ; z=\mathrm{i} a \frac{\mathrm{e}+\mathrm{e}^{\mathrm{i} \theta}}{\mathrm{e}-\mathrm{e}^{\mathrm{i} \theta}}, \theta \in[0,2 \pi]\right\} \leq C N^{-1} .
$$

Then for any $K>0$, there is $N_{0}=N_{0}(a, C, K)$ such that for $N \geq N_{0}$,

$$
\begin{aligned}
& \sup \left\{\left|m_{\mu_{N}-\mu}(E+\mathrm{i} \eta)\right| ; E \in[-K, K], \frac{16}{\sqrt{a \log (N / C)}} \leq \eta \leq a \frac{\mathrm{e}-1}{\mathrm{e}+1}\right\} \\
& \quad \leq \mathrm{e}^{-8 \sqrt{\log (N / C) / a}} .
\end{aligned}
$$

PROOF. We apply the previous theorem: here, $c=4 / a, \delta=C N^{-1}$, so that $r(\delta)=\mathrm{e}^{-8 / \sqrt{a \log (N / C)}}$. Thus $1-r(\delta) \leq 8 / \sqrt{a \log (N / C)}$ and it is easy to see that for $N$ large enough, the disc $H_{a, r(\delta)}$ contains the set in question here.

Acknowledgements. We would like to thank the referees for their careful readings of our paper and for all the remarks and suggestions.

\section{REFERENCES}

[1] Anderson, G. W., Guionnet, A. and Zeitouni, O. (2010). An Introduction to Random Matrices. Cambridge Studies in Advanced Mathematics 118. Cambridge Univ. Press, Cambridge. MR2760897

[2] BAO, Z., ERDős, L. and SCHNELLI, K. (2016). Local stability of the free additive convolution. J. Funct. Anal. 271 672-719. MR3506962

[3] BAO, Z., ERDős, L. and SchNELLI, K. Local law of addition of random matrices on optimal scale. Preprint. Available at arXiv:1509.07080.

[4] BASAK, A. and Dembo, A. (2013). Limiting spectral distribution of sums of unitary and orthogonal matrices. Electron. Commun. Probab. 18 no. 69, 19. MR3091727

[5] BelinsCHI, S. T. (2008). The Lebesgue decomposition of the free additive convolution of two probability distributions. Probab. Theory Related Fields 142 125-150. MR2413268

[6] Belinschi, S. T. (2014). $L^{\infty}$-boundedness of density for free additive convolutions. Rev. Roumaine Math. Pures Appl. 59 173-184. MR3299499

[7] Belinschi, S. T., Benaych-Georges, F. and Guionnet, A. (2009). Regularization by free additive convolution, square and rectangular cases. Complex Anal. Oper. Theory 3 611-660. MR2551632

[8] Belinschi, S. T. and BERCOVICI, H. (2007). A new approach to subordination results in free probability. J. Anal. Math. $101357-365$. 
[9] Belinschi, S. T., Bercovici, H., Capitaine, M. and FÉvrier, M. Outliers in the spectrum of large deformed unitarily invariant models. Preprint. Available at arXiv:1207.5443.

[10] BenAyCh-Georges, F. (2009). Rectangular random matrices, related convolution. Probab. Theory Related Fields 144 471-515. MR2496440

[11] BENAYCH-GEORGES, F. (2015). Exponential bounds for the support convergence in the single ring theorem. J. Funct. Anal. 268 3492-3507. MR3336731

[12] Benaych-Georges, F. and Knowles, A. Lectures on the local semicircle law for Wigner matrices. Preprint. Available at arXiv:1601.04055.

[13] Bercovici, H. and Voiculescu, D. (1998). Regularity questions for free convolution. In Nonselfadjoint Operator Algebras, Operator Theory, and Related Topics. Oper. Theory Adv. Appl. 104 37-47. Birkhäuser, Basel. MR1639647

[14] Biane, P. (1998). Processes with free increments. Math. Z. 227 143-174. MR1605393

[15] Bordenave, C. and Chafaï, D. (2012). Around the circular law. Probab. Surv. 9 1-89. MR2908617

[16] Bourgade, P., Yau, H.-T. and Yin, J. (2014). The local circular law for random matrices. Probab. Theory Related Fields 159 545-595.

[17] Bourgade, P., YAu, H.-T. and Yin, J. (2014). The local circular law II: The edge case. Probab. Theory Related Fields 159 619-660. MR3230004

[18] Chafaï, D., Guédon, O., Lecué, G. and Pajor, A. (2012). Interactions Between Compressed Sensing Random Matrices and High Dimensional Geometry. Panoramas et Synthèses 37. Société Mathématique de France, Paris.

[19] Erdôs, L., Schlein, B. and YAU, H.-T. (2009). Semicircle law on short scales and delocalization of eigenvectors for Wigner random matrices. Ann. Probab. 37 815-852. MR2537522

[20] Erdős, L., RAmírez, J., Schlein, B. and YAU, H.-T. (2010). Universality of sine-kernel for Wigner matrices with a small Gaussian perturbation. Electron. J. Probab. 15 526-603.

[21] Ferreira, O. P. and Svaiter, B. F. Kantorovich's theorem on Newton's method. Preprint. Available at arXiv:1209.5704.

[22] Guionnet, A., Krishnapur, M. and Zeitouni, O. (2011). The single ring theorem. Ann. of Math. (2) 174 1189-1217. MR2831116

[23] Guionnet, A. and Zeitouni, O. (2012). Support convergence in the single ring theorem. Probab. Theory Related Fields 154 661-675. MR3000558

[24] HaAgerup, U. and LARSEN, F. (2000). Brown's spectral distribution measure for $R$-diagonal elements in finite von Neumann algebras. J. Funct. Anal. 176 331-367. MR1784419

[25] Hardy, G. H. (1915). The mean value of the modulus of ananalytic function. Proc. Lond. Math. Soc. (3) 14 269-277.

[26] Kargin, V. (2012). A concentration inequality and a local law for the sum of two random matrices. Probab. Theory Related Fields 154 677-702. MR3000559

[27] Kargin, V. (2013). An inequality for the distance between densities of free convolutions. Ann. Probab. 41 3241-3260. MR3127881

[28] Kargin, V. (2015). Subordination of the resolvent for a sum of random matrices. Ann. Probab. 43 2119-2150.

[29] Ledoux, M. (2001). The Concentration of Measure Phenomenon. Mathematical Surveys and Monographs 89. Amer. Math. Soc., Providence, RI. MR1849347

[30] NiCA, A. and SPEICHER, R. (2006). Lectures on the Combinatorics of Free Probability. London Mathematical Society Lecture Note Series 335. Cambridge Univ. Press, Cambridge. MR2266879

[31] Rudelson, M. and Vershynin, R. (2014). Invertibility of random matrices: Unitary and orthogonal perturbations. J. Amer. Math. Soc. 27 293-338. MR3164983

[32] TAO, T. and VU, V. (2010). Random matrices: Universality of ESDs and the circular law. Ann. Probab. 38 2023-2065. 
[33] Voiculescu, D. V., Dykema, K. J. and NicA, A. (1992). Free Random Variables. CRM Monograph Series 1. Amer. Math. Soc., Providence, RI. MR1217253

[34] YIN, I. (2014). The local circular law III: General case. Probab. Theory Related Fields 160 679-732.

MAP 5, UMR CNRS 8145

UNIVERSITÉ PARIS DESCARTES

45 RUE DES SAINTS-PÈRES 75270

PARIS CEDEX 6

FRANCE

E-MAIL: florent.benaych-georges@parisdescartes.fr 